\title{
A Statistical Theory of the Electromagnetic Field Polarization Parameters at the Scattering by Distributed Radar Objects
}

\author{
Victor Tatarinov and Sergey Tatarinov \\ Tomsk State University of Control Systems and Radioelectronics \\ Russian Federation
}

\section{Introduction}

The development of coherent radars leads to discovery of coherent images specific properties at the electromagnetic waves scattering random distributed radar objects (RDRO). It was fixed that these images are having a stochastic - spotty structure. This structure is stipulated by interference of waves scattered by a random collection of RDRO secondary radiation centers. The similar phenomenon in optics has named as "speckles". So far all existent investigations both in the optic area and in RDRO coherent images for microwaves area (speckles theory) were made only for the case of electromagnetic waves linear polarization. However, the results of scattered wave's polarization parameters extensive experimental investigations at the scattering by man - made RDRO have demonstrated the existence of speckle - effect for scattered field polarization parameters. One from main aspects of a developed polarization parameters statistical theory appears an establishment of connection between RCRO coherent images polarization parameters with these objects space spectra. It is demonstrated that a formation process of RDRO coherent images polarization - energetically parameters can be considered as the interference process. A validity of emergence principle for polarization - energetically parameters at the scattering by RDRO has demonstrated. A polarization coherence notion has introduced.

A generalization of Fresnel - Arago interference laws has demonstrated an emergence of a new physical effect, which demonstrates that at the scattering by RDRO a scattered field polarization - energetically parameters are not defined by an union of RDRO separate scatterers polarization parameters. How it is demonstrated, in the reality these parameters are defined be relations between RDRO separate scatterers properties.

As far as polarization - energetically parameters of RDRO coherent images are having the intensity dimension, then RCRO random polarization - energetically responses autocorrelation functions (ACF) will be as correlations of the 4-th order. It is demonstrated for the first time that ACF of the 4-th degree and a distribution of polarization proximity (distance) along a RDRO space spectra are related by Fourier transformations pair. Thus, a connection between scattered field polarization energetically parameters distribution and polarization parameters distribution along a RDRO space spectrum can be found. 


\section{Electromagnetic waves scattering by random distributed object}

\subsection{Scattering geometry for random distributed object}

A considerable number of articles are devoted to radar objects (RO) scattering properties. So, the scattering properties of RO having the simplest form were analyzed in «Proceedings of the IEEE» (1965. Vol. 53, № 8) and in the book (Ufimtsev, 1963). The special issues of «Proc. of the IEEE» (1985, Vol. 77, № 5), and «IEEE Trans. on Antennas and Propagation» (1989, № 5) were devoted to complex radar objects scattering properties. At this case complex radar objects were defined as non-regular bodies in contrast with simplest objects, which are usual body of rotation. However, all these investigations did not take into account a polarization of electromagnetic field. Complex (distributed) $\mathrm{RO}$ can be presented also as a combination of "point" scatterers (point RO) (Ostrovityanov, 1982), (Shtager, 1994). It is necessary to point out into attention that the notion of a "brilliant" point (or secondary center of radiation), which is defined by Fresnel the first zone is well known an it is used in radar theory at the definition of a radar object cross section ((RCS). For the correct definition of radiation secondary center we will use the expression for a radar object cross section in the form

$$
\sigma=\frac{\pi}{\lambda^{2}}\left|\int \dot{I}(z) \exp \left\{j 2 k_{0} z \cos \frac{\beta}{2}\right\} d z\right|,
$$

which is valid both two-position and one-position radar (Kell, 1965). The expression (1) is one from the forms of Stratton-Chu integral (Stratton \& Chu, 1939) for the electromagnetic wave scattering by object having an arbitrary form . Here value $\beta$ is two-positional scattering angle. The $\mathrm{OZ}$ axis is coinciding with this angle bisector. A vectorial function $\dot{I}(z)$ is connected with electrical and magnetic vectors of an incident wave. The integral (1) can be decomposed to an union of integrals. Every from these integrals is integrated into an area $z$, where integrand function is continuous. All these integrals can be interpreted as a "simplest" center of secondary radiation (Kell, 1965), which is stipulated by a stationary phase area . A contribution of every secondary radiation center in full diffraction integral (1) is connected with a stationary phase area size, i.e. an area near considered radiation center. Into the limits of this area a summarized phase of integrand function $\dot{I}(z) \exp \left\{j 2 k_{0} z \cos \beta / 2\right\}$ is differing not more than $\pi / 2$ from its value in the central point of secondary radiation area.

If a radar object will have only one area (center) of secondary radiation, it will be an onepoint radar object. In this case an unique are of secondary radiation must have a fixed phase center. An object area, which is defined a scattered field can be as ideal conducting surface or it can be having an electric anisotropy (Kell, 1965).

An electric anisotropy property of a secondary radiation area is defined the connection between of incident and scattered waves electric vectors $\dot{\vec{E}}_{I}$ in the form of matrix equation, which for the case of one-position radar has the form [Tatarinov et al, 2006]

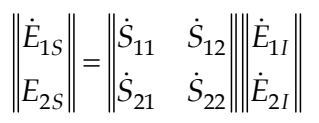

where the matrix $\left\|\dot{S}_{j l}\right\|(j, l=1,2)$ is so-called back-scattering matrix (or scattering matrix) of a point scattering radar object. 
Let us consider that a random complex radar object (RDRO) is a system of $N$ rigidly connected scattering centers $T_{M},(M=1, \ldots N)$ each having local scattering matrix $\left\|\dot{S}_{j l}^{m}\right\|$. All scattering centers are contained in area T. The outline of this area is the outline of the RDRO ( see fig. 1 ).

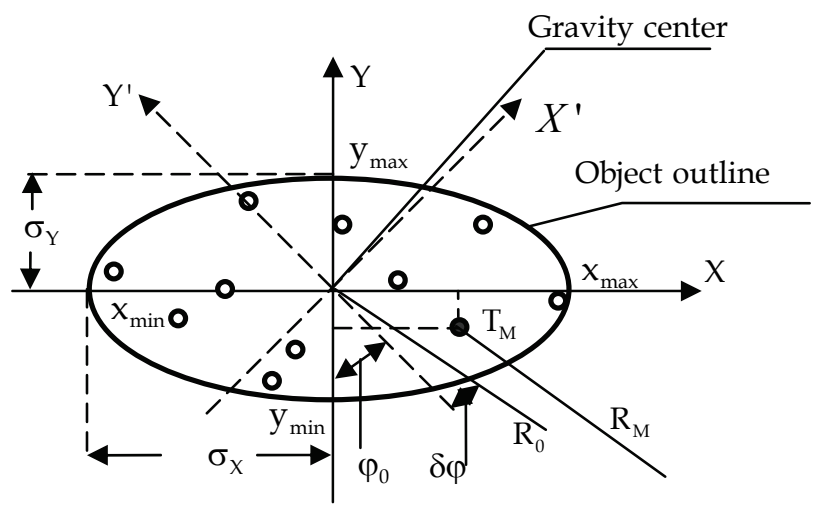

Fig. 1. The scattering geometry

Every realization of such a random system is a collection of a large number $N$ of points reflectors (scattering centers). The $M$-th scattering center has a position $x_{M}, \mathrm{y}_{M}$ in the coordinate system XOY connected with the object. The origin of coordinates is the "center of gravity" of the reflecting system. The conditions $\sum_{M=1}^{N} x_{M}=0 ; \quad \sum_{M=1}^{N} y_{M}=0$ are existing in this connection. The values $\sigma_{X}, \sigma_{Y}$ are essential mean square of the random reflecting system sizes and these values can be written as $\sigma_{X}=\sqrt{(1 / N-1) \sum_{M=1}^{N} x_{M}^{2}}$;

$$
\begin{gathered}
\sigma_{Y}=\sqrt{(1 / N-1) \sum_{M=1}^{N} y_{M}^{2}} \text {. For the case } N>1 \text { the approximation } \\
\sigma_{X}=\sqrt{(1 / N) \sum_{M=1}^{N} x_{M}^{2}} ; \quad \sigma_{Y}=\sqrt{(1 / N) \sum_{M=1}^{N} y_{M}^{2}}
\end{gathered}
$$

will be correct. Then mean square sizes of the distributed reflecting system along essential directions $O X, O Y$ can be defined in the form

$$
L_{X}=2 \sqrt{(1 / N) \sum_{M=1}^{N} x_{M}^{2}} ; \quad L_{Y}=2 \sqrt{(1 / N) \sum_{M=1}^{N} y_{M}^{2}} .
$$

If the probability density of reflectors coordinates is uniform, we can write (Kobak, 1975)

$$
L_{X}=\left(x_{\max }-x_{\min }\right) / \sqrt{3} ; \quad L_{Y}=\left(y_{\max }-y_{\min }\right) / \sqrt{3}
$$


The distributed (complex) radar object representation in the form of scattered centers collection can be confirmed by the experimental results.

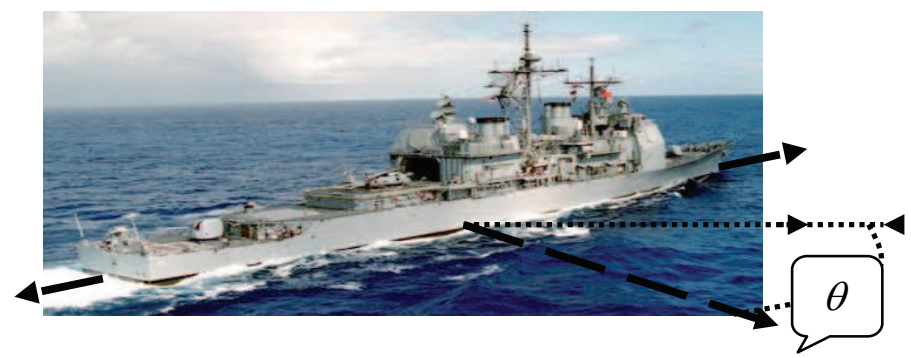

Fig. 2. The sea ship picture

So, on fig. 2 the picture of a sea ship is shown and on fig. 3 the optical simulation results of electromagnetic waves scattering by this ship are placed (Shtager, 1994). On this picture a lot of brilliant points are represented. These points are secondary scattering centers. A random collection of brilliant points is characterizing an electromagnetic field scattered both by complex (distributed) radar object and by the sea surface.

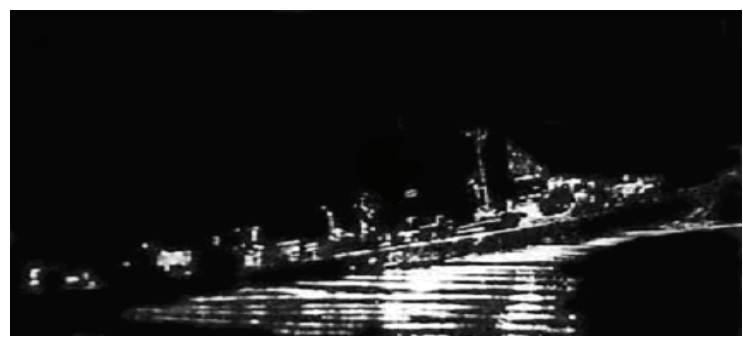

Fig. 3. The optical simulation results of electromagnetic waves scattering by the ship

Modern methods of complex radar objects experimental investigations allows us to have the sizes of the resolution cell equal to $1 \times 1 \mathrm{~m}$. In this case the resolution along the range is provided using ultra short pulses (5-7 nanosecond) and angular resolution is provided by aperture synthesis. This experimental radar has been designed in USA (Steinberg, 1989).. The fig. 4 presents two-dimensional radar map of the plane Locheed L - 1011 that was obtained with the use radar mentioned and its picture. It follows from these pictures that scattering centers distribution correctly represents the plane structure.
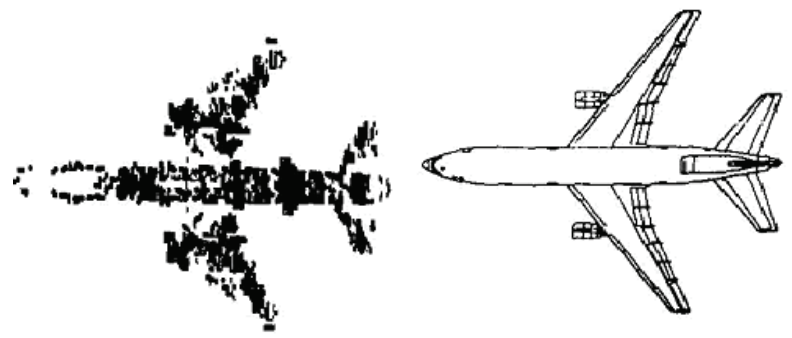

Fig. 4. The secondary scattering centers of the plane Locheed L - 1011 and its picture 
Let us now to find a distance $R_{M}$ between a scatterer $T_{M}$ and radar, taking into account that a positional angle $\varphi$ of object has defined in the form $\varphi=\varphi_{0} \pm \delta \varphi$, where $\varphi_{0}$ is a mean positional angle. In what follows we will define a scattered field into some angular interval $\pm \delta \varphi$ relative to angle $\varphi_{0}$. In far zone a distance $R_{M}$ can be found in the form

$$
R_{M}=R_{0}-\left[\left(x_{M} \cos \varphi_{0}-y_{M} \sin \varphi_{0}\right) \sin \delta \varphi+\left(y_{M} \cos \varphi_{0}+x_{M} \sin \varphi_{0}\right) \cos \delta \varphi\right] .
$$

Using the approximation $\sin \delta \varphi \approx \delta \varphi, \cos \delta \varphi \approx 1$ we see that the expression (6) will have the form $R_{M}=R_{0}-\left[x_{M}^{\prime} \delta \varphi+y^{\prime}{ }_{M}\right]$, where $x_{M}^{\prime}=x_{M} \cos \varphi_{0}-y_{M} \sin \varphi_{0}, y^{\prime}{ }_{M}=y_{M} \cos \varphi_{0}+x_{M} \sin \varphi_{0}$ are Cartesian coordinates of $M$ - th scattering center into new coordinate system $X^{\prime} 0 Y^{\prime}$ that is rotated on an angle $\varphi_{0}$ relative to initial coordinate system XOY (see fig. 1). With regard to statistical independence of values $x_{M}, y_{m}$ we can find mean square sizes of distributed radar object into new coordinate system $X^{\prime} 0 Y^{\prime}$ in the form

$$
L_{X}^{\prime}=\sqrt{0,5\left(L_{X}^{2}+L_{Y}^{2}\right)} \sqrt{1+\gamma_{T} C_{2 \varphi 0}} ; L_{Y}^{\prime}=\sqrt{0,5\left(L_{X}^{2}+L_{Y}^{2}\right)} \sqrt{1-\gamma_{T} C_{2 \varphi 0}}
$$

Here and later on the designation $C_{\varphi} \equiv \cos \varphi ; S_{\varphi} \equiv \sin \varphi$ for the writing shortening are used. Into the expressions (7) the designation $\gamma_{T}=\left(L_{X}^{2}-L_{Y}^{2}\right) /\left(L_{X}^{2}+L_{Y}^{2}\right)$ was introduced, where values $L_{X}, L_{Y}$ are mean square sizes of distributed radar object into its initial coordinate system XOY. So, a value $\gamma_{T}$ is dimensionless factor for characteristic of distributed radar object on the average. We will name this factor as "distributed radar object geometric anisotropy degree".

\subsection{Polarization invariants of distributed object elementary scatterer and their geometric representation}

For the definition of an electromagnetic field at the scattering by random distributed object we must determine of polarization parameters system for description of scattering process by elementary (point) scatterer in the best way.

Let us to write an electromagnetic field, scattered by point scattering center $T_{M}$ in the form

$$
\dot{\vec{E}}_{S}^{M}(\omega)=\frac{\exp \left(-j 2 k R_{M}\right)}{R_{M} \sqrt{4 \pi}}\left\|S_{j l}^{M}\right\| \overrightarrow{\vec{E}}_{0}
$$

taking into account the coherent nature of scattering process. Here a value $R_{M}$ is the distance between scatterer $T_{m}$ and radar; matrix $\left\|S_{. j l}^{M}\right\|$ is a scattering matrix of distributed radar object elementary scatterer; vectors $\dot{\vec{E}}_{0}$ and $\overrightarrow{\vec{E}}_{S}$ are complex Jones vectors of radiated and scattered waves respectively. Now we will analyze most important invariant of elementary scatterer scattering matrix - a complex degree of point radar object polarization anisotropy. For this parameter introduction we will use the writing of radar object scattering matrix in the Cartesian polarization basis under the condition of arbitrary angle of object eigencoordinates system and radar coordinates system mutual orientation

$$
\left\|\dot{S}_{j l}\right\|=\left\|\begin{array}{cc}
C_{\beta} & -S_{\beta} \\
S_{\beta} & C_{\beta}
\end{array}\right\|\left\|\begin{array}{ll}
\dot{\lambda}_{1} & 0 \\
0 & \lambda_{2}
\end{array}\right\|\left\|C_{\beta} \quad S_{\beta}\right\|=0,5\left\|\begin{array}{cc}
\left(\dot{\lambda}_{1}+\dot{\lambda}_{2}\right)+\left(\dot{\lambda}_{1}-\dot{\lambda}_{2}\right) C_{2 \beta} & \left(\dot{\lambda}_{1}-\dot{\lambda}_{2}\right) S_{2 \beta} \\
\left(\dot{\lambda}_{1}-\dot{\lambda}_{2}\right) S_{2 \beta} & \left(\dot{\lambda}_{1}+\dot{\lambda}_{2}\right)-\left(\dot{\lambda}_{1}-\dot{\lambda}_{2}\right) C_{2 \beta}
\end{array}\right\| .
$$


Here $\beta$ is mutual orientation angle, $\dot{\lambda}_{1}=\lambda_{1} \exp \{i \varphi / 2\}, \dot{\lambda}_{2}=\lambda_{2} \exp \{-i \varphi / 2\}$ are scattering matrix complex eigenvalues, $\lambda_{1}, \lambda_{2}$ are their modules and $\varphi$ is arguments difference. Let us decompose this matrix using Pauli matrix system:

$$
\left\|\dot{S}_{j l}\right\|=0,5\left\{S_{0}^{S M}\left\|\begin{array}{cc}
1 & 0 \\
0 & 1
\end{array}\right\|+S_{1}^{S M}\left\|\begin{array}{cc}
1 & 0 \\
0 & -1
\end{array}\right\|+S_{2}^{S M}\left\|\begin{array}{cc}
0 & 1 \\
1 & 0
\end{array}\right\|+S_{0}^{S M}\left\|\begin{array}{cc}
0 & i \\
-i & 0
\end{array}\right\|\right\},
$$

where $S_{j}^{S M}=S p\left\{\left\|\sigma_{j}\right\|\left\|\dot{S}_{j l}\right\|\right\},(j=0,1,2,3)$ are developing coefficients. So,we can rewrite the expression $(8 b)$ in the form

$$
\left\|S_{j l}\right\|=0,5\left(\dot{\lambda}_{1}+\dot{\lambda}_{2}\right)\left\{\left\|\begin{array}{cc}
1 & 0 \\
0 & 1
\end{array}\right\|+\dot{\mu}\left\|\begin{array}{cc}
\cos 2 \beta & \sin 2 \beta \\
\sin 2 \beta & -\cos 2 \beta
\end{array}\right\|\right\},
$$

where the complex value

$$
\dot{\mu}=\left(\dot{\lambda}_{1}-\dot{\lambda}_{2}\right) /\left(\dot{\lambda}_{1}+\dot{\lambda}_{2}\right)
$$

is the "complex degree of the polarization anisotropy" (CDPA). It follows from this expression that a scattering matrix $\left\|S_{j l}\right\|$ can be presented in the weighted sum of an isotropic radar object, i.e. trihedral corner reflector and dihedral corner reflector, a weight of which is defined by $\dot{\mu}$ value. Let us write the CDPA module as follows

$$
|\dot{\mu}|=\sqrt{\dot{\mu} \dot{\mu}^{*}}=\sqrt{\left(\lambda_{1}^{2}+\lambda_{2}^{2}-2 \lambda_{1} \lambda_{2} C_{\varphi}\right) / \lambda_{1}^{2}+\lambda_{2}^{2}+2 \lambda_{1} \lambda_{2} C_{\varphi}} .
$$

The real part, imaginary part and argument of the CDPA can be written in the form

$$
\operatorname{Re}\{\dot{\mu}\}=\frac{\lambda_{1}^{2}-\lambda_{2}^{2}}{\lambda_{1}^{2}+\lambda_{2}^{2}+2 \lambda_{1} \lambda_{2} C_{\varphi}} ; \operatorname{Im}\{\dot{\mu}\}=\frac{2 \lambda_{1} \lambda_{2} S_{\varphi}}{\lambda_{1}^{2}+\lambda_{2}^{2}+2 \lambda_{1} \lambda_{2} C_{\varphi}} ; \arg \{\dot{\mu}\}=\arctan \left(\frac{2 \lambda_{1} \lambda_{2} S_{\varphi}}{\lambda_{1}^{2}-\lambda_{2}^{2}}\right),
$$

where $\lambda_{1}$ and $\lambda_{2}$ are the modules of the eigen values; $\varphi=\arg \left\{\dot{\lambda}_{1}\right\}-\arg \left\{\dot{\lambda}_{2}\right\}$ is the arguments' difference corresponding to the phase shift caused by the object' electric properties. It is obvious that the values $|\dot{\mu}|, \operatorname{Re}\{\dot{\mu}\}, \operatorname{Im}\{\dot{\mu}\}, \arg \{\dot{\mu}\}$ are also the invariants of the scattering matrix. Thus, the CDPA fully describes the polarization properties of the object. The introduction of the CDPA notion allows presenting on the complex plane the objects' polarization properties how it was demonstrated in (Tatarinov at al, 2006).

Let us consider this aspect more explicitly. It can be shown with the use of expressions (11) and (12) that $\dot{\mu}$-value can be presented on the complex plane $\dot{\mu}=\operatorname{Re}\{\dot{\mu}\}+j \operatorname{Im}\{\dot{\mu}\}$ (fig.5). The points of this plane are connected with the polarization properties of the radar objects as far as $\dot{\mu}$-value is a radar object scattering matrix polarization invariant. The origin of the coordinates $(\dot{\mu}=0)$ corresponds to the object characterizing by values $\lambda_{1}=\lambda_{2}, \varphi=0$ (a phase shift between eigenvalues is zero). It follows from the necessity to simultaneously satisfy the conditions $\operatorname{Re}\{\dot{\mu}\}=0, \quad \operatorname{Im}\{\dot{\mu}\}=0$. The physical analog of this point is polarization isotropic radar object (trihedral corner reflector, sphere, metal plate ). The point at infinity corresponds to the object having the following polarization properties: 
$\lambda_{1}=\lambda_{2}, \varphi= \pm \pi$. The physical analog of the point at infinity is the dihedral corner reflector. All points of the imaginary axis correspond to the objects with $\operatorname{Re}\{\dot{\mu}\}=0$, i.e. $\lambda_{1}=\lambda_{2}$. In this case, the points laying on the positive imaginary semi-axis, present radar objects which are characterized by the phase shift $\varphi>0$, while the negative imaginary semi-axis

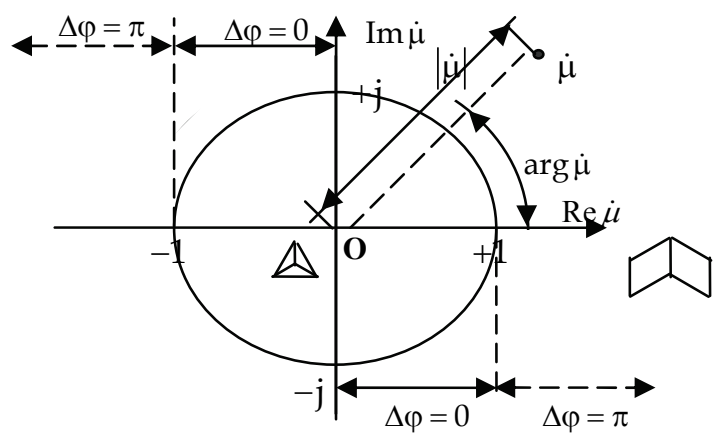

Fig. 5. The complex plane of radar objects

$(\operatorname{Im}\{\dot{\mu}\}<0)$ depicts the objects with $\varphi<0$. The points $\pm j$ present the objects having $\varphi= \pm(\pi / 2)$ phase shift. All real axis's points of the complex $\dot{\mu}$-plane correspond to the objects with zero phase shift $\varphi=0$; (i. e. $\operatorname{Im}\{\dot{\mu}\}=0)$. However, the given case is complicated by the fact that the object, which corresponds to the point at infinity, is the dihedral reflector. This contradiction can be solved, considering the equality $\sin \varphi=0$ both for $\varphi=0$; and $\varphi=\pi$; cases. Then the points of the real axis of the complex $\dot{\mu}$-plane must be determined with the use of the conditions $(\cos 0=1, \cos \pi=-1)$ as $\operatorname{Re}\{\dot{\mu}\}=\left(\lambda_{1}^{2}-\lambda_{2}^{2}\right) /\left(\lambda_{1}^{2}+\lambda_{2}^{2}+2 \lambda_{1} \lambda_{2} C_{\varphi}\right)$. Thus, into the interval $\operatorname{Re}\{\dot{\mu}\}=0 ; \operatorname{Re}\{\dot{\mu}\}=1$ the value $\lambda_{2}$ reduces from $\lambda_{1}=\lambda_{2}$ in the origin up to $\lambda_{2}=0$ in the point $\operatorname{Re}\{\dot{\mu}\}=1$ (horizontal oriented object). This point depicts the "degenerated" radar object (long linear object, dipole, i.e. polarizer). The phase shift in the point has an undefined value. It changes spasmodically by $\pi$ when passing the point $\operatorname{Re}\{\dot{\mu}\}=1$. Then, the value $\lambda_{2}$ increases from $\lambda_{2}=0$ (in the point $\operatorname{Re}\{\dot{\mu}\}=1)$ up to $\lambda_{1}=\lambda_{2}$ (the point at infinity). In this case, the phase shift along the ray $\operatorname{Re}\{\dot{\mu}\}=1 ; \operatorname{Re}\{\dot{\mu}\}=\infty$ equals to $\pi$. The similar analysis can be made with respect to the negative semi-axis $\operatorname{Re}\{\dot{\mu}\}$. Thus, the complex $\dot{\mu}$-plane has the properties equivalent to the properties of the circular complex plane. However, at that time when the circular complex plane presents the polarization properties of electromagnetic waves, the complex $\dot{\mu}$-plane is intended for presentation of the invariant polarization parameters of the radar objects scattering matrix.

Analyzing the similarity, which exists between the $\dot{\mu}$-plane and the circular complex plane, we can conclude that it is expedient to choose the circular basis as the basis for presenting the radiated and scattered waves. The scattering matrix (8) in the circular basis can be found in the form (Tatarinov at al, 2006) 


$$
\left\|S_{j l}^{R L}\right\|=0,5 j\left\|\begin{array}{cc}
\left(\dot{\lambda}_{1}-\dot{\lambda}_{2}\right) \exp \{j(2 \beta-\pi / 2)\} & \left(\dot{\lambda}_{1}+\dot{\lambda}_{2}\right) \\
\left(\dot{\lambda}_{1}+\dot{\lambda}_{2}\right) & \left(\dot{\lambda}_{1}-\dot{\lambda}_{2}\right) \exp \{-j(2 \beta-\pi / 2)\}
\end{array}\right\| .
$$

Change of the rotation direction under backscattering is also considered in this expression. Let us present the radiated wave in the circular basis $\left(\dot{\vec{e}}_{R}, \dot{\vec{e}}_{L}\right)$. The circular polarization ratio for this wave can be written as $\dot{P}_{R}^{R L}=\dot{E}_{R} / \dot{E}_{L}$. Then, the circular polarization ratio for the scattered wave will have the form

$$
\dot{P}_{S}^{R L}=\left\{1+\dot{\mu} \exp [-j(2 \beta-\pi / 2)] \dot{P}_{R}^{R L}\right\} /\left\{\dot{\mu} \exp [-j(2 \beta-\pi / 2)]+\dot{P}_{R}^{R L}\right\} .
$$

It is possible to set the specific polarization state of the radiated wave, when polarization ratio of the scattered wave will have an unique form. So, if $\dot{P}_{R}^{R L}=\infty$, (right circular polarized wave ) then we can rewrite the expression (14) in the form

$$
\dot{P}_{S}^{R L}=\lim _{\dot{P}_{R}^{R L} \rightarrow \infty} \frac{1+\dot{\mu} \exp \{-j(2 \beta-\pi / 2)\} \dot{P}_{R}^{R L}}{\dot{\mu} \exp \{-j(2 \beta-\pi / 2)\}+\dot{P}_{R}^{R L}} \exp \{-j[2 \beta-\arg (\dot{\mu})-\pi / 2]\} .
$$

If $\theta=0$, then we get

$$
\dot{P}_{S}^{R L}=|\dot{\mu}| \exp \{-j[\arg (\dot{\mu})+\pi / 2]\} .
$$

Using the Jones vector $\dot{\vec{E}}_{R L}$ we can find the circular polarization ratio in the form

$$
\dot{P}^{R L}=\tan (\alpha+\pi / 4) \exp \{-i(2 \beta-\pi / 2)\} .
$$

Here $\alpha$ is an ellipticity angle and $\beta$ is an orientation angle of polarization ellipse.

The comparison of expressions $(16 \mathrm{a}, \mathrm{b})$ shows that the measured module of the circular polarization ratio of the scattered wave (when the radiated wave has right circular polarization) is equal to the complex degree polarization anisotropy (CDPA) module

$$
\left|\dot{P}_{S}^{R L}\right|=\tan (\alpha+\pi / 4)=|\dot{\mu}| .
$$

The argument of the $\dot{P}_{S}^{R L}$ (for the case $\theta=0$ ) can be presented as $\arg \{\dot{\mu}\}+\pi / 2=-2 \beta+\pi / 2$ or $\arg \{\dot{\mu}\}=-2 \beta$. The last expression demonstrates that the value of CDPA argument determines the orientation of the polarization ellipse in the eigencoordinates system of the scattering object. If $\theta \neq 0$, then the polarization ellipse will be rotated additionally an angle of $2 \beta$. The correspondence between the circular complex plane and the Riemann sphere, having unit diameter, was analyzed in details in (Tatarinov at al, 2006) with the use of the stereographic projection equations, which are connecting onto-one the circular complex plane points $\dot{P}^{R L}=\operatorname{Re} \dot{P}^{R L}+j \operatorname{Im} \dot{P}^{R L}$ with Cartesian coordinates $X_{1}, X_{2}, X_{3}$ of the point $S$, laying on the Riemann sphere surface. The transition from the circular complex plane to the Poincare sphere, having unit radius, can be realized with the use the modified stereographic projection equations 


$$
X=2 \operatorname{Re} \dot{P}^{R L} /\left(1+\left|\dot{P}^{R L}\right|^{2}\right), \quad Y=2 \operatorname{Im} \dot{P}^{R L} /\left(1+\left|\dot{P}^{R L}\right|^{2}\right), Z=2\left\{\left|\dot{P}^{R L}\right|^{2} /\left(1+\left|\dot{P}^{R L}\right|^{2}\right)-0,5\right\} .
$$

Using these equations we can connect the complex $\dot{\mu}$-plane of radar objects with the sphere of unit radius(fig. 6).

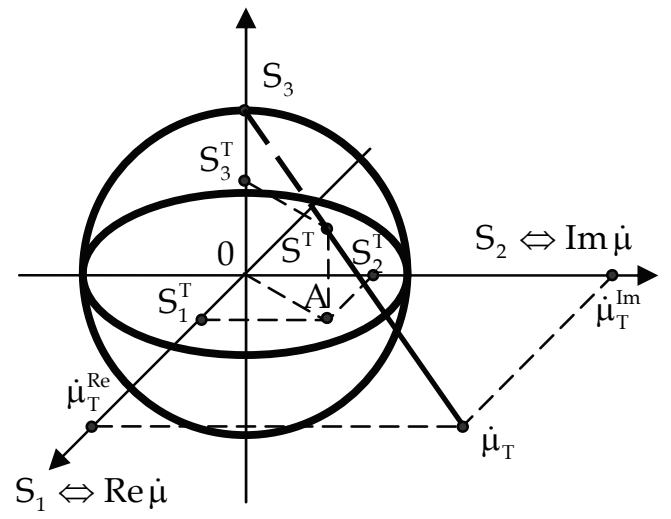

Fig. 6. Polarization sphere of radar objects

We will assume that the axes $\vec{S}_{1}, \vec{S}_{2}$ of the three-dimensional space $S_{1}, S_{2}, S_{3}$ are coinciding with real and imaginary axes $\operatorname{Re}\{\dot{\mu}\}, \operatorname{Im}\{\dot{\mu}\}$ of the radar objects complex plane $\dot{\mu}=\operatorname{Re} \dot{\mu}+j \operatorname{Im} \dot{\mu}$ respectively. In accordance with stated above, all point of this $S_{T}$-sphere will be connected one-to-one with corresponding points of radar objects complex $\dot{\mu}$-plane. Let us consider now that a radar object is defined on the $\dot{\mu}$-plane by the point $\dot{\mu}_{T}=\mu_{R}+j \mu_{I}$. Then we will connect the point $\dot{\mu}_{T}$ with the sphere north pole by the line, which crosses the sphere surface at point $S^{T}$. The projections $S_{1}^{T}, S_{2}^{T}, S_{3}^{T}$ of the point $S_{T}$ to the axes $S_{1}, S_{2}, S_{3}$ will be defined to modified stereographic projection equation. It is not difficult to see that these values are satisfying to the unit sphere equation $\left(S_{1}^{T}\right)^{2}+\left(S_{2}^{T}\right)^{2}+\left(S_{3}^{T}\right)^{2}=1$.

Thus, all points of the complex plane of radar objects are corresponding one-to-one to points of the sphere $S_{T}$. We will name this sphere as the unit sphere of radar object.

\subsection{Scattering operator of distributed radar object and its factorization}

Let us to write now the Jones vector of the field scattered by the RDRO in the form

$$
\left\|\bar{E}_{S \Sigma}(k, \delta \varphi)\right\|=\frac{\exp \left\{-j 2 k R_{0}\right\}}{R_{0} \sqrt{4 \pi}}\left\|\begin{array}{ll}
\sum_{M=1}^{N} \dot{S}_{11}^{M} \exp \left\{j 2 k \eta^{M}\right\} & \sum_{M=1}^{N} \dot{S}_{12}^{M} \exp \left\{j 2 k \eta^{M}\right\} \|{\dot{\dot{E}_{1}^{0}}}_{\sum_{M=1}^{N} \dot{S}_{21}^{M} \exp \left\{j 2 k \eta^{M}\right\}} \sum_{M=1}^{N} \dot{S}_{22}^{M} \exp \left\{j 2 k \eta^{M}\right\}
\end{array}\right\|,
$$

where $\eta^{m}=x_{m}^{\prime} \delta \varphi+z_{m}^{\prime}$ and matrix 


$$
\left\|\dot{S}_{j l}^{\Sigma}(k, \delta \varphi)\right\|=\frac{\exp \left\{-j 2 k R_{0}\right\}}{R_{0} \sqrt{4 \pi}}\left\|\begin{array}{ll}
\sum_{M=1}^{N} \dot{S}_{11}^{M} \exp \left\{j 2 k \eta^{M}\right\} & \sum_{M=1}^{N} \dot{S}_{12}^{M} \exp \left\{j 2 k \eta^{M}\right\} \\
\sum_{M=1}^{N} \dot{S}_{21}^{M} \exp \left\{j 2 k \eta^{M}\right\} & \sum_{M=1}^{N} \dot{S}_{22}^{M} \exp \left\{j 2 k \eta^{M}\right\}
\end{array}\right\|
$$

is scattering operator, which includes space, polarization and frequency property of random distributed radar object. It follows from the expression (19) that all elements of the RDRO scattering operator $\left\|S_{j l}^{\Sigma}(k, \delta \varphi)\right\|$ are the function of two variables. These variables are as the wave vector absolutely value and positional angle $\varphi$. A dependence from the wave vector absolutely value is the frequency dependence, as far as for a media having the refraction parameter $n=1$ the wave vector has the form $k=2 \pi / \lambda=\omega / c$, where $c$ is light velocity and $\omega=2 \pi f$. It is necessary to note here that scattered field polarization parameters at the scattering by one-point radar object are independent both from positional angle and frequency. For analysis of polarization-angular and polarization-frequency dependences of the field at the scattering by the RDRO we write the exponential function $\exp \left\{-j\left[2 k x_{M}^{\prime} \delta \varphi+2 k z_{M}^{\prime}\right]\right\}$ that has been included into the operator (19) elements. The index of this function is originated by the existence both angular and frequency dependences of the field scattered by the RDRO. We will rewrite this index for its analysis:

$$
f_{M}(k, \delta \varphi)=2 k x_{M}^{\prime} \delta \varphi+2 k z_{M}^{\prime} .
$$

Let's us assume that the initial wave is quasimonochromatic $\left(\Delta \omega / \omega_{0}<<1\right)$ and that radar radiation frequency arbitrary changes are not disturbing this condition. We can write the wave vector $k$ absolutely value in the form

$$
k=(\omega / c)=\left(\omega_{0}+\Delta \omega\right) / c,
$$

where $\omega_{0}$ is a mean constant frequency of radar radiation, and $\Delta \omega$ is a variable part originated by radar radiation frequency change or frequency modulation. The substitution of the expression (21) in the expression (20) give us

$$
\begin{aligned}
& f_{M}(k, \delta \varphi)=2\left[\left(\omega_{0}+\Delta \omega\right) x_{m}^{\prime} \delta \varphi / c\right]+2\left[\left(\omega_{0}+\Delta \omega\right) z_{M}^{\prime} / c\right]= \\
& =2\left(\omega_{0} x_{M}^{\prime} \delta \varphi / c\right)+2\left(\omega_{0} z_{M}^{\prime} / c\right)+2\left(x_{M}^{\prime} \delta \varphi \Delta \omega+z_{M}^{\prime} \Delta \omega\right) / c
\end{aligned} .
$$

As far as the value $\omega_{0}$ is constant, then from all items of the expression (22) only the value $2 x_{m}^{\prime} \delta \varphi \Delta \omega / c$ is depending simultaneously both on variable positional angle $\delta \varphi$ and on frequency variable $\Delta \omega$. However, it is not difficult to see that the inequality

$$
2\left(x_{M}^{\prime} \delta \varphi \Delta \omega / c\right)<<2\left(z_{M}^{\prime} \Delta \omega / c\right)
$$

is correct under the condition $\delta \varphi<<1 \operatorname{Rad}$ (i.e. $\delta \varphi \leq 10^{\circ}$ ). Taking into account this inequality, we can neglect by the value $2 x_{M}^{\prime} \delta \varphi \Delta \omega / c$ in the equation (23) and then we can rewrite it in the form

$$
f_{M}\left(k_{0}, \delta \varphi, \Delta \omega\right) \approx 2 k_{0} x_{M}^{\prime} \delta \varphi+t_{M}^{\prime} \omega
$$


where $\omega=\omega_{0}+\Delta \omega, t_{M}^{\prime}=2 z_{M}^{\prime} / c$. Thus, the angular and frequency variables in the expression (24) are separated. It is so-called factorization operation. The value $t_{M}$ is a doubled time interval, which is necessary for initial wave passage of a distance, which is a projection of segment $z_{M}$ on the $O Z^{\prime}$ axis, i.e. on the propagation direction of radar initial wave. This analysis shows that the scattered field polarization parameters frequency dependence at the scattering by the RCRO is defined by the projections of the scattering centers co-ordinates on the OZ' axis, which is coinciding with the radar initial wave propagation direction. In other words, a frequency dependence is defined by the RCRO extension along the initial wave propagation direction. It follows simultaneously from the equation (33) that the scattered field polarization-angular dependence on the mean frequency $\omega_{0}$ is defined by the values $x_{m}^{\prime}$ collection. These values are projections of scattering centers positions on the $O X^{\prime}$ axis that is perpendicular to radar initial wave propagation direction. So, an extension of the RCRO along the $O X^{\prime}$ axis is originated a polarization-angular dependence of field polarization parameters at the scattering by a RCRO.

\section{Angular response function of a distributed object and its basic forms}

Taking into account the results of subsection 2.3 we can now consider separately the polarization-angular and polarization-frequency forms of a distributed radar object responses on unit action, having circular polarization.

In accordance with the mentioned results the polarization-angular response of a complex object at mean frequency $\omega_{0}$ is determined by extension of the object along the axis $O X^{\prime}$, that is perpendicular to direction of incident wave propagation's. Taking into account the expression (18) we can write the scattering operator (28) of the distributed radar object for the circular polarization basis in the form

$$
\left\|\dot{S}_{j l}^{\Sigma, R L}\left(k_{0}, \delta \varphi\right)\right\|=\frac{\exp \left\{-j 2 k_{0} R_{0}\right\}}{R_{0} \sqrt{4 \pi}}\left\|\begin{array}{lll}
\dot{S}_{11}^{\Sigma, R L}\left(k_{0}, \delta \varphi\right) & \dot{S}_{12}^{\Sigma, R L}\left(k_{0}, \delta \varphi\right) \\
\dot{S}_{21}^{\Sigma, R L}\left(k_{0}, \delta \varphi\right) & \dot{S}_{22}^{\Sigma, R L}\left(k_{0}, \delta \varphi\right)
\end{array}\right\|,
$$

Where

$$
\begin{aligned}
& \dot{S}_{11}^{\Sigma, R L}\left(k_{0}, \delta \varphi\right)=\sum_{M=1}^{N}\left|\dot{\Delta}^{M}\right| \exp \left\{j\left[2 k_{0} x_{M}^{\prime} \delta \varphi-\beta_{3 M}\right]\right\} ; \dot{S}_{22}^{\Sigma, r l}\left(k_{0}, \delta \varphi\right)=-\sum_{m=1}^{N}\left|\dot{\Delta}^{m}\right| \exp \left\{j\left[2 k_{0} x_{m}^{\prime}-\beta_{1 m}\right]\right\} \\
& \dot{S}_{12}^{\Sigma, r l}\left(k_{0}, \delta \varphi\right)=\dot{S}_{21}^{\Sigma, r l}\left(k_{0}, \delta \varphi\right)=j \sum_{m=1}^{N}\left|\dot{\Sigma}^{m}\right| \exp \left\{j\left[2 k_{0} x_{m}^{\prime} \delta \varphi-\beta_{2 m}\right]\right\} .
\end{aligned}
$$

Here $\beta_{2 M}=\arg \dot{\Sigma}^{M}-2 k z_{M}^{\prime} ; \beta_{1 M}=\arg \dot{\Delta}^{M}-2 \theta_{M}-2 k z_{M}^{\prime} ; \beta_{2 M}=\arg \dot{\Sigma}^{M}-2 k z_{M}^{\prime}$; $\beta_{3 M}=\arg \dot{\Delta}^{M}+2 \theta_{M}-2 k z_{M}^{\prime}$ and values $\dot{\Delta}^{\mathrm{M}}=\dot{\lambda}_{1}^{\mathrm{M}}-\dot{\lambda}_{2}^{\mathrm{M}}, \dot{\Sigma}^{M}=\dot{\lambda}_{1}^{M}+\dot{\lambda}_{2}^{M}$ are the difference and union of $M$-th elementary scatterers eigen values. If the Jones vector of the incident wave is right circular polarized, we can write for the circular Jones vector of the wave, scattered by a distributed radar object in the form 


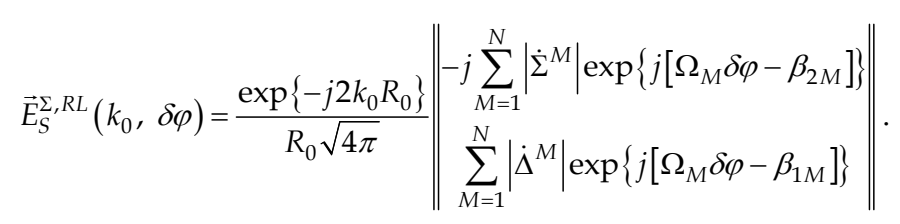

We are using here the notion of spatial frequencies $\Omega_{M}=2 k_{0} x_{M}^{\prime}$ (Kobak, 1975), (Tatarinov et al, 2006) that allows us to consider the elements of the Jones vector (26) as the sum of a large number harmonic oscillations. The moving coordinate of these oscillation is the variable positional angle $\delta \varphi$. The frequencies of these oscillations are determined by projections of the coordinates of scattering centers $T_{M}$ on the axis $O X^{\prime}$.

Amplitudes of oscillations are the values $\left|\dot{\Sigma}^{M}\right|, \dot{\Delta}^{M}$ can be characterized by the Rayleigh distribution (Potekchin et al, 1966) and the random initial phases $\beta_{1 M}, \beta_{2 m}$ may have the uniform distribution into the interval $0 \div 2 \pi$. Stochastic values of the spatial frequencies $\Omega_{M}$ may have the uniform distribution in the interval $\Omega_{M I N} \div \Omega_{M A X}$. This interval correspond to domain of definition $x_{M I N}^{\prime} \div x_{M A X}^{\prime}$ along the $O X^{\prime}$ axis. Thus, we can consider the sum (26) as a complex stochastic function of the moving coordinate $\delta \varphi$.

The circular polarization ratio for mean frequency $\omega_{0}$ we can write using elements of Jones vector (26) in this case will have the form

$$
\dot{P}_{S}^{R L}\left(k_{0}, \delta \varphi\right)=j \sum_{M=1}^{N}\left|\dot{\Delta}^{M}\right| \exp \left\{j\left[\Omega_{M} \delta \varphi-\beta_{1 M}\right]\right\} / \sum_{M=1}^{N}\left|\dot{\Sigma}^{M}\right| \exp \left\{j\left[\Omega_{M} \delta \varphi-\beta_{2 M}\right]\right\} .
$$

This ratio represents an angular distribution of the polarization parameters of an RCRO and it is the polarization-angular response function of a random distributed radar object on the unit action, having the form of a circular polarized wave.

Polarization-angular response function (27) is a generalization of the point object response (16a) on the unit action, having the form of a circular polarized wave. Both the polarization properties of scatterers, and geometrical parameters of a random distributed radar object are represented into the polarization-angular response (27). We will transform every item of the numerator of (27) in the following form

$$
\begin{gathered}
\left|\dot{\Delta}^{M}\right| \exp \left\{j\left[\Omega_{M} \delta \varphi-\beta_{1 M}\right]\right\}=\left|\dot{\Sigma}^{M}\right|\left(\left|\dot{\Delta}^{M}\right| /\left|\dot{\Sigma}^{M}\right|\right) \exp \left\{j\left[\Omega_{M} \delta \varphi-\beta_{1 M}\right]\right\}= \\
=\left|\dot{\Sigma}^{M}\right|\left|\dot{\mu}^{M}\right| \exp \left\{j\left[\Omega_{M} \delta \varphi-\beta_{1 M}\right]\right\}
\end{gathered}
$$

Here the values $\left|\dot{\mu}^{M}\right|$ are determined by expression (10) and represent modules of elementary scatterer's $T_{M}$ complex degree polarization anisotropy. The values $\left|\dot{\mu}^{M}\right|$, that are describing the polarization properties of elementary reflectors of an RDRO, make up a general expression by using the weight factors $\left|\dot{\Sigma}^{M}\right|=\left[\left(\lambda_{1}^{M}\right)^{2}+\left(\lambda_{2}^{M}\right)^{2}+2 \lambda_{1}^{M} \lambda_{2}^{M} \cos \Delta \varphi_{M}\right]^{0,5}$. Taking into account this fact, we can find 


$$
\dot{P}_{S}^{R L}\left(k_{0}, \delta \varphi\right)=j \sum_{M=1}^{N}\left|\dot{\Sigma}^{M}\right|\left|\dot{\mu}^{M}\right| \exp \left\{j\left(\Omega_{M} \delta \varphi-\beta_{1 M}\right)\right\} / \sum_{M=1}^{N}\left|\dot{\Sigma}^{M}\right| \exp \left\{j\left(\Omega_{M} \delta \varphi-\beta_{2 M}\right)\right\} .
$$

The weight factors $\left|\dot{\Sigma}^{m}\right|$ are connected with the radar cross sections of elementary scatterers. The angular distribution of the polarization ratio (28) completely describes the polarization structure of the field, scattered by a complex object

$$
\dot{P}_{S}^{R L}\left(k_{0}, \delta \varphi\right)=\tan \left[\alpha\left(k_{0}, \delta \varphi\right)+\pi / 4\right] \exp \left\{j 2 \beta\left(k_{0}, \delta \varphi\right)\right\} .
$$

Here values $\alpha\left(k_{0}, \delta \varphi\right)$ and $\beta\left(k_{0}, \delta \varphi\right)$ are angular distributions both of the ellipticity angle and the orientation angle of the polarization ellipse of the scattered field.

Existing measurement methods allow us to carry out direct measurements of the module of a polarization ratio. Thus, we have the possibility for the direct measurements of ellipticity angle of the scattered wave. The measurement of the orientation needs indirect methods.

First of all we shall consider the opportunity of the characteristics of an ellipticity angle in the analysis of wave polarization, scattered by random distributed objects. We will use all forms of complex radar object polarization-angular response, which are different functions of an ellipticity angle. The following parameters are connected with an ellipticity angle value:

- The value $\tan (\alpha+\pi / 4)=\left|\dot{P}^{R L}\left(k_{0}, \delta \varphi\right)\right|$, determined in the interval

$$
0 \leq \tan (\alpha+\pi / 4) \leq \infty \text {; }
$$

- The coefficient of ellipticity $K\left(k_{0}, \delta \varphi\right)=\tan \alpha$, determined in the interval $-1 \leq \tan \alpha \leq 1$. This coefficient is connected with the module of circular polarization ratio $\left|\dot{P}^{R L}\right|$ by

$$
K\left(k_{0}, \delta \varphi\right)=\tan \alpha=\left[\left(\left|\dot{P}^{R L}\right|-1\right) /\left(\left|\dot{P}^{R L}\right|+1\right)\right]=[\tan (\alpha+\pi / 4)-1] /[\tan (\alpha+\pi / 4)+1]
$$

- -The third normalized Stokes parameter $S_{3}=\sin 2 \alpha$, determined in the interval $-1 \leq S_{3} \leq 1$.

This parameter is connected with the square of the circular polarization ratio module as

$$
S_{3}\left(k_{0}, \delta \varphi\right)=\sin 2 \alpha\left(k_{0}, \delta \varphi\right)=\left[\left|\dot{P}_{S}^{R L}\left(k_{0}, \delta \varphi\right)\right|^{2}-1\right] /\left[\left|\dot{P}_{S}^{R L}\left(k_{0}, \delta \varphi\right)\right|^{2}+1\right] .
$$

The Stokes parameter $S_{3}$ and the ellipticity coefficient $K$ are connected by the expression

$$
S_{3}=\sin 2 \alpha=\sin (2 \arctan K)=2 K /\left(1+K^{2}\right) .
$$

The inverse function $K\left(S_{3}\right)$ is the solution of the equation $S_{3} K^{2}-2 K+S_{3}=0$. We will choose the solution $K\left(S_{3}\right)=\left(1-\sqrt{1-S_{3}^{2}}\right) / S_{3}$ from two versions $K_{1 / 2}=\left(1 \pm \sqrt{1-S_{3}^{2}}\right) / S_{3}$. It follows from conformity $K=-1, S_{3}=-1 ; K=0, S_{3}=0 ; K=1, S_{3}=1$ that only solution 
(4c) remains. Thus, we can use the initial polarization-angular response $\left|\dot{P}_{S}^{r l}\left(k_{0}, \delta \varphi\right)\right|=\tan \left[\alpha\left(k_{0}, \delta \varphi\right)+\pi / 4\right]$ and two other forms of responses $K\left(k_{0}, \delta \varphi\right)=\tan \left[\alpha\left(k_{0}, \delta \varphi\right)\right]$ and $S_{3}\left(k_{0}, \delta \varphi\right)=\sin 2 \alpha\left(k_{0}, \delta \varphi\right)$.

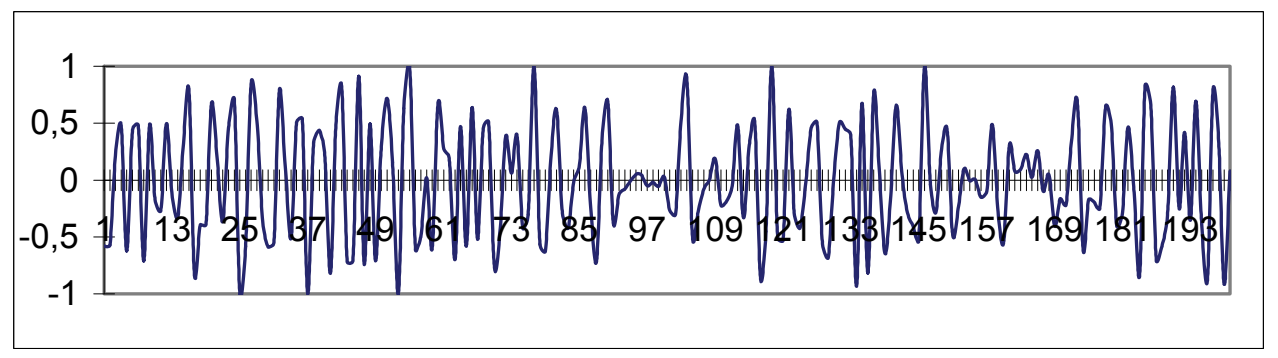

Fig. 7. The experimental realization of polarization- angular response function $S_{3}(\delta \varphi)$

For example, the experimental realization, having the form of narrow-band angular dependence $S_{3}(\delta \varphi)$ has shown on the Fig. 7. The angular extension of this experimental realization is $\pm 20^{\circ}$ at the observation to radar object board. The samples of polarizationangular response function are following with the angular interval $0,2^{0}$.

\section{An emergence principle and polarization coherence notion}

The analysis of an electromagnetic field polarization properties at the scattering by space distributed radar object is closely connected with two key problems. The first problem is the influence of separated scatterers space diversity on scattered field polarization. The second key problem of polarization properties investigation at the scattering by distributed radar object is connected with scattered field polarization properties definition on the base of the emergence principle with the use of possible relations between complex radar object parts polarization properties.

\subsection{An emergence principle and space frequency notion for a simplest distributed object. polarization proximity and polarization distance}

Let us to define a field, scattered by RDO using the Stratton-Chu integral (1), which allows us to represent this field as the union of waves scattered by elementary scatterers ("bright" or "brilliant" points), forming complex object. For the case when every elementary scatterer is characterizing by its scattering matrix $\left\|S_{j l}^{M}\right\| ;(i, 1=1,2)$ then the scattered field complex vector can be defined in the form

$$
\left\|\dot{\vec{E}}_{S}^{\Sigma}\left(k_{0}, \delta \varphi\right)\right\|=\frac{\exp \left\{-j 2 k_{0} R_{0}\right\}}{R_{0} \sqrt{4 \pi}} \sum_{M=1}^{N}\left\|S_{j l}^{M}\right\|\left\|\overrightarrow{\vec{E}}_{0}\right\|,
$$

where $R_{0}$ is a distance between the radar and object gravity center, $\delta \varphi$ is a positional angle of the object and $\left\|\overrightarrow{\vec{E}}_{0}\right\|$ is the complex vector of initial wave. It is necessary to indicate here that the expression (32) has been represented only individual polarization properties of 
every from scatterers, which are forming a large distributed radar object. Unfortunately, a large system property in principle can not be bringing together to an union of this system elements properties. The conditionality of integral system properties appear by means of its elements relations. These relations lead to the "emergence" of new properties which could not exist for every element separately. The emergence notion is one from main definitions of the systems analysis (Peregudov \& Tarasenko, 2001). Let us consider the simplest distributed radar object in the form of two closely connected scatterers $A$ and $B$ (reflecting elliptical polarizers), which can not be resolved by the radar. These scatterers are distributed in the space on the distance $l$ and are characterizing by the scattering matrices in the Cartesian polarization basis:

$$
\left\|S_{1}\right\|=\left\|\begin{array}{cc}
\dot{a}_{1} & 0 \\
0 & \dot{a}_{2}
\end{array}\right\|,\left\|S_{2}\right\|=\left\|\begin{array}{cc}
\dot{b}_{1} & 0 \\
0 & \dot{b}_{2}
\end{array}\right\| .
$$

It will be the case of coherent scattering and its geometry is shown on the fig. 8 .

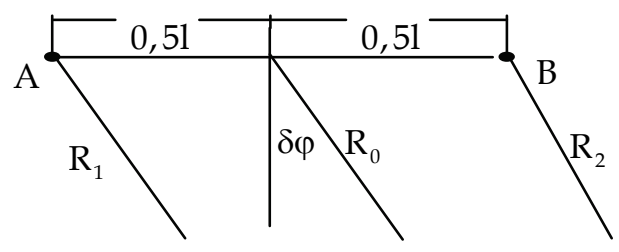

Fig. 8. The scattering by two-point radar object

Here the distances $R_{1}, R_{2}$ between the scatterers and arbitrary point $Q$ in far zone can be written in the form $R_{1,2} \approx R_{0} \pm 0,5 l \sin \delta \varphi \approx R_{0} \pm 0,5 l \delta \varphi$ under the condition $0,5 l<<R_{0}$. Using these expressions, we can find the Jones vector of the scattered field for the case when radiated signal has linear polarization $45^{\circ}$

$$
\dot{\vec{E}}_{S}(\delta \varphi)=\frac{\sqrt{2}}{2}\left\|\begin{array}{l}
\dot{a}_{1} \exp (j \xi)+\dot{b}_{1} \exp (-j \xi) \\
\dot{a}_{2} \exp (j \xi)+\dot{b}_{2} \exp (-j \xi)
\end{array}\right\|,
$$

where $\xi=k l \theta$. Let us to define now a polarization- energetical response functions in the form of Stokes momentary parameters $S_{0}, S_{3}$ angular dependences

$$
S_{0}(\delta \varphi)=\dot{E}_{X}(\delta \varphi) \dot{E}_{X}^{*}(\delta \varphi)+\dot{E}_{Y}(\delta \varphi) \dot{E}_{Y}^{*}(\delta \varphi) ; S_{3}(\delta \varphi)=i\left[\dot{E}_{X}(\delta \varphi) \dot{E}_{Y}^{*}(\delta \varphi)-\dot{E}_{Y}(\delta \varphi) \dot{E}_{X}^{*}(\delta \varphi)\right] .
$$

The expanded form of the energetically response function $S_{0}(\delta \varphi)$ can be found as

$$
S_{0}(\delta \varphi)=0,5\left[S_{0}^{A}+S_{0}^{B}\right]+\sqrt{a_{1}^{2} b_{1}^{2}+a_{2}^{2} b_{2}^{2}+\dot{a}_{1}^{*} \dot{a}_{2} \dot{b}_{1} \dot{b}_{2}^{*}+\dot{a}_{1} \dot{a}_{2}^{*} \dot{b}_{1}^{*} \dot{b}_{2}} \cos \left(2 \xi+\eta_{1}\right)
$$

where $\eta_{1}=\arctan \left\{\left[\operatorname{Im}\left(\dot{a}_{1} \dot{b}_{1}^{*}+\dot{a}_{2} \dot{b}_{2}^{*}\right) / \operatorname{Re}\left(\dot{a}_{1} \dot{b}_{1}^{*}+\dot{a}_{2} \dot{b}_{2}^{*}\right)\right]\right\}$ and $S_{0}^{A}=a_{1}^{2}+a_{2}^{2}, \quad S_{0}^{B}=b_{1}^{2}+b_{2}^{2}$. The values $S_{0}^{A}, S_{0}^{B}$ are the Stokes zero-parameters of elementary scatterers $A$ and $B$. The polarization-angular response function $S_{3}(\delta \varphi)$ has the form 


$$
S_{3}(\delta \varphi)=0,5\left[S_{3}^{A}+S_{3}^{B}\right]+2 \sqrt{a_{1}^{2} b_{1}^{2}+a_{2}^{2} b_{2}^{2}-\left(\dot{a}_{1}^{*} \dot{a}_{2} \dot{b}_{1} \dot{b}_{2}^{*}+\dot{a}_{1} \dot{a}_{2}^{*} \dot{b}_{1}^{*} \dot{b}_{2}\right)} \sin \left(2 \xi+\eta_{2}\right),
$$

where $\quad \eta_{2}=\arctan \left\{\left[\operatorname{Im}\left(\dot{a}_{1} \dot{b}_{2}^{*}-\dot{a}_{2} \dot{b}_{1}^{*}\right) / \operatorname{Re}\left(\dot{a}_{1} \dot{b}_{2}^{*}-\dot{a}_{2} \dot{b}_{1}^{*}\right)\right]\right\} \quad$ and $\quad S_{3}^{A}=-05 j\left(\dot{a}_{1} \dot{a}_{2}^{*}-\dot{a}_{1}^{*} \dot{a}_{2}\right)$, $S_{3}^{B}=-0,5 j\left(\dot{b}_{1} \dot{b}_{2}^{*}-\dot{b}_{1}^{*} \dot{b}_{2}\right)$ are the 3-rd Stokes parameters of elementary scatterers $A$ and $B$.

The angular harmonic functions $\cos \left[2 k l \delta \varphi+\eta_{1}\right], \sin \left[2 k l \theta+\eta_{2}\right]$ in the expressions $(35 \mathrm{a}, \mathrm{b})$, are representing the influence of scatterers $A$ and $B$ space diversity to the scattered field polarization-energetically parameters distribution in far zone. The derivative from angular harmonic functions full phases $\psi(\delta \varphi)=2 k l \delta \varphi+\eta_{k}(k=1,2)$ along the angular variable is the space frequency $f_{S P}=(1 / 2 \pi)(d / d \delta \varphi)\left[2 k l \delta \varphi+\eta_{k}\right]=2 l / \lambda$.

Now we will analyze the amplitudes of angular harmonic functions $\cos \left[2 k l \delta \varphi+\eta_{1}\right]$, $\sin \left[2 k l \delta \varphi+\eta_{2}\right]$. Let us write first of all the polarization rations $\dot{P}_{A}=\dot{a}_{2} / \dot{a}_{1}$ and $\dot{P}_{B}=\dot{b}_{2} / \dot{b}_{1}$ which are characterizing the point radar objects $A$ and $B$ on the complex plane of radar objects. We can find the spherical distance between the points $S_{A}, S_{B}$, laying on the surface of the Riemann sphere having unit diameter, which are connected with points $\dot{P}_{A}, \dot{P}_{B}$ of radar objects complex plane. The coordinates of the points $S_{A}, S_{B}$ on the sphere surface are $X_{1}=\operatorname{Re} \dot{P} /\left(1+|\dot{P}|^{2}\right) ; \quad X_{2}=\operatorname{Im} \dot{P} /\left(1+|\dot{P}|^{2}\right) ; \quad X_{3}=|\dot{P}|^{2} /\left(1+|\dot{P}|^{2}\right)$ and a spherical distance between these points can be found in the form (Tatarinov et al, 2006)

$\rho_{S}\left(S_{A}, S_{B}\right)=\left|\dot{P}_{A}-\dot{P}_{B}\right| / \sqrt{1+\left|\dot{P}_{A}\right|^{2}} \sqrt{1+\left|\dot{P}_{B}\right|^{2}},(36)$ where $\left|\dot{P}_{A}-\dot{P}_{B}\right|$ is the Euclidian metric on the complex plane of radar objects. After substitution of the polarization ratios $\dot{P}_{A}=\dot{a}_{2} / \dot{a}_{1}$ and $\dot{P}_{B}=\dot{b}_{2} / \dot{b}_{1}$ into the expressions (46) we can write

$$
\rho_{S}\left(S_{A}, S_{B}\right)=\frac{\sqrt{\left|\dot{P}_{A}\right|^{2}+\left|\dot{P}_{B}\right|^{2}-\left(\dot{P}_{A} \dot{P}_{B}^{*}+\dot{P}_{A}^{*} \dot{P}_{B}\right)}}{\sqrt{1+\left|\dot{P}_{A}\right|^{2}} \sqrt{1+\left|\dot{P}_{B}\right|^{2}}}=\sqrt{\frac{a_{1}^{2} b_{1}^{2}+a_{2}^{2} b_{2}^{2}-\left(\dot{a}_{1}^{*} \dot{a}_{2} \dot{b}_{1} \dot{b}_{2}^{*}+\dot{a}_{1} \dot{a}_{2}^{*} \dot{b}_{1}^{*} \dot{b}_{2}\right)}{\left(a_{1}^{2}+a_{2}^{2}\right)\left(b_{1}^{2}+b_{2}^{2}\right)}},
$$

where the value

$$
D=\left[a_{1}^{2} b_{1}^{2}+a_{2}^{2} b_{2}^{2}-\left(\dot{a}_{1}^{*} \dot{a}_{2} \dot{b}_{1} \dot{b}_{2}^{*}+\dot{a}_{1} \dot{a}_{2}^{*} \dot{b}_{1}^{*} \dot{b}_{2}\right)\right] /\left(a_{1}^{2}+a_{2}^{2}\right)\left(b_{1}^{2}+b_{2}^{2}\right)
$$

is so-called polarization distance between two waves (or radar objects polarization states), having different polarizations (Azzam \& Bashara, 1980), (Tatarinov et al, 2006). It is not difficult to demonstrate that the waves having coinciding polarizations $\left(\dot{P}_{A}=\dot{P}_{B}\right)$ are having the polarization distance value $D=0$ and the waves having orthogonal polarizations $\left(\dot{P}_{B}=-1 / \dot{P}_{A}^{*}\right)$ have the polarization distance value $D=1$. Thus, it follows from (37) and (38) that $a_{1}^{2} b_{1}^{2}+a_{2}^{2} b_{2}^{2}-\left(\dot{a}_{1}^{*} \dot{a}_{2} \dot{b}_{1} \dot{b}_{2}^{*}+\dot{a}_{1} \dot{a}_{2}^{*} \dot{b}_{1}^{*} \dot{b}_{2}\right)=D\left(a_{1}^{2}+a_{2}^{2}\right)\left(b_{1}^{2}+b_{2}^{2}\right)$.

We can use also so-called polarization proximity value $N=1-D$. Using values $N, D$ we can rewrite the expressions $(35 a, b)$ in the form

$$
S_{0}(\delta \varphi)=0,5\left[S_{0}^{A}+S_{0}^{B}+2 \sqrt{S_{0}^{A}} \sqrt{S_{0}^{B}} \sqrt{N} \cos \left(2 \xi+\eta_{1}\right)\right] .
$$




$$
S_{3}(\delta \varphi)=0,5\left[S_{3}^{A}+S_{3}^{B}+2 \sqrt{S_{0}^{A}} \sqrt{S_{0}^{B}} \sqrt{D} \sin \left(2 \xi+\eta_{2}\right)\right] .
$$

We can consider these expressions as generalized interference laws as far as these expression are the generalization of Fresnel-Arago interference laws (Tatarinov et al, 2007). It follows from the expression (39) that the orthogonal polarized waves can not give an interference picture, as far as for the polarization proximity value $N=0$. However, the expression (40) demonstrates that in this case we will have the maximal value of this interference picture visibility. It follows from expressions (40) that for every Stokes parameters have the place some constant component, which is defined by the according Stokes parameters of both objects $(A$ and $B)$, and space harmonics function $\cos \left[2 k l \delta \varphi+\eta_{1}\right], \quad \sin \left[2 k l \delta \varphi+\eta_{2}\right]$, having amplitudes $2 \sqrt{S_{0}^{A}} \sqrt{S_{0}^{B}} \sqrt{N}, 2 \sqrt{S_{0}^{A}} \sqrt{S_{0}^{B}} \sqrt{D}$ and space initial phase $\eta_{k}$. So, the polarization-energetically properties of complex radar object can not be found only with the use of its elements properties. The conditionality of integral system properties appear by means of its elements relations. These relations in our case are polarization distance and polarization proximity. The use of these values leads to the "emergence" of new properties which did not exist for every element separately.

\subsection{A polarization coherence notion and its definition as the correlation moment of the forth order}

Let us to define a momentary visibility of generalized interference law (39) in the form

$$
W=\left[S_{0}^{M A X}(\theta)-S_{0}^{M I N}(\theta)\right] /\left[S_{0}^{M A X}(\theta)+S_{0}^{M I N}(\theta)\right]=2 \sqrt{S_{0}^{A}} \sqrt{S_{0}^{B}} \sqrt{N} /\left(S_{0}^{A}+S_{0}^{B}\right) .
$$

The equation (41) is coinciding with well known expression for partial coherent field interference law visibility (Born \& Wolf, 1965 ), (Potekchin \& Tatarinov, 1978)

$$
W=\left[I^{M A X}(\theta)-I^{M I N}(\theta)\right] /\left[I^{M A X}(\theta)+I^{M I N}(\theta)\right]=2 \sqrt{I_{1}} \sqrt{I_{2}} \gamma_{12} /\left[I_{1}+I_{2}\right],
$$

where $I_{1}, I_{2}$ are power of waves summarized and $\gamma_{12}$ is a coherence degree. If $I_{1}=I_{2}$ then an interference law visibility is defined by coherence degree having the second order.

So, we can claim, that from physical point of view the parameter $N$ can be considered as polarization coherence parameter, which defines a proximity of elementary scatterers polarization states, analogously coherence degree of stochastic waves summarized. In this case we have "momentary" value of polarization coherence, at the some time a coherence degree $\gamma_{12}$ is the correlation value. In this connection it is necessary to analyze statistical effects and polarization coherence mean value.

If we will consider the interference law (39) visibility, then we can see that it is defined by a value $\sqrt{N}$, which is a magnitude of space harmonic function $\cos \left[2 k l \delta \varphi+\eta_{1}\right]$. It is necessary to point out that a value $\sqrt{N}$ is corresponding to polarization coherence of the second order. However, it is clear that value $N$ is corresponding to polarization coherence of the forth order. On the fig. 9 the interference law (39) is presented for the case $S_{0}^{A}=S_{0}^{B}$. In this case the interference law visibility is defined by value $\sqrt{N}$. 


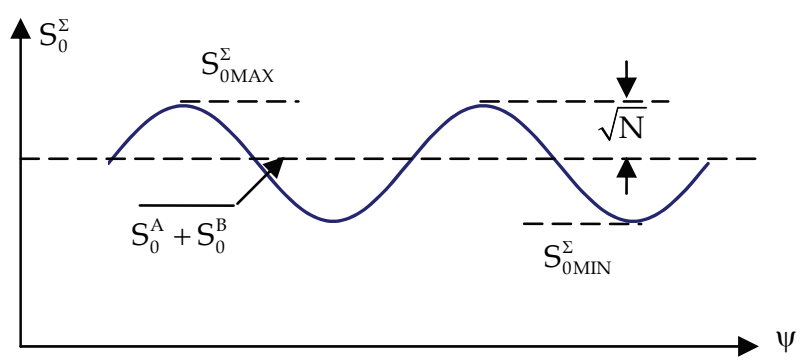

Fig. 9. To polarization coherence definition

A magnitude of space harmonic function $\sin \left[2 k l \delta \varphi+\eta_{2}\right]$ into the interference law (40) for the third Stokes parameter is defined by elementary scatterers $A$ and $B$ polarization states distance $\sqrt{D}$. As far as $\sqrt{D}=\sqrt{1-N}$, then a value $\sqrt{D}$ can be considered also as polarization coherence of the second order.

It follows from the expressions $(39,40)$ that polarization states proximity and distance are included into the interference laws in the form $\sqrt{N}$ and $\sqrt{D}$. It provides power dimension for these laws. Let us to find now an autocovariance function of the interference law (39) for polarization coherence mean value definition. We will assume here that space harmonics amplitudes $\sqrt{N}$ and space initial phase $\eta$ are random independent variables. For this case their two-dimensional probability distribution can be presented as two one-dimensional distributions densities product $W_{2}(\sqrt{N}, \eta)=W_{1}(\sqrt{N}) W_{1}(\eta)$. We will assume also that $W(\eta)=1 / 2 \pi)$. We can presuppose also that $S_{0}^{A}=S_{0}^{B}=S_{0}$. At that time auto covariance function can be defined in the form of the mean value

$$
K_{S}^{0}(\Delta \varphi)=S_{0}^{2}\left\{1+\overline{N \cos \left[2 k l \delta \varphi+\eta_{1}\right] \cos \left[2 k l(\delta \varphi+\Delta \varphi)+\eta_{1}\right]}\right\}=S_{0}^{2}\left[1+B_{S}^{0}(\Delta \varphi)\right]
$$

that is statistical moment of the forth order. Here the function $B_{S}^{0}(\Delta \theta)$ is the autocorrelation function of scattered field intensity

$$
B_{S}^{0}(\Delta \varphi)=\int_{0}^{\infty} \int_{-\infty}^{\infty}(\sqrt{N})^{2} \cos \left[2 k l \delta \varphi+\eta_{1}\right] \cos \left[2 k l(\delta \varphi+\Delta \varphi)+\eta_{1}\right] W_{1}(\sqrt{N}) W_{1}(\eta) d(\sqrt{N}) d \eta .
$$

The integration of this expression gives us

$$
B_{S}^{0}(\Delta \varphi)=\frac{0,5}{2 \pi} \int_{0}^{\infty} \int_{-\pi}^{\pi}(\sqrt{N})^{2} \cos (2 k l \Delta \varphi) W_{1}(\sqrt{N}) d(\sqrt{N}) d \eta=0,5 \bar{N} \cos (2 k l \Delta \varphi),
$$

where $\bar{N}$ is the mean value of elementary scatterers $A$ and $B$ polarization states proximity. It is defined amplitudes of space harmonics collection having $f_{S P}=2 l / \lambda$.

Thus, both autocovariance function and autocorrelation function are correlation function of the forth order and they are describing the intensity correlation for interference law (39). In this connection autocovariance function (42) is the interference law of the forth order. A visibility of this law is defined by polarization coherence degree $\bar{N}$. 
For the interference law (40) under the condition $S_{0}^{A}=S_{0}^{B}=S_{0}$ autocovariance function has the form

$$
K_{S}^{3}(\Delta \varphi)=0,25 S_{0}^{2}\left\{\left[\left(S_{3}^{\Sigma}\right)^{2} / S_{0}^{2}\right]+2 B_{S}^{3}(\Delta \varphi)\right\}
$$

and it is (how earlier) statistical moment of the forth order. Here $S_{3}^{\Sigma}=S_{3}^{A}+S_{3}^{B}$, and function $B_{S}^{3}(\Delta \varphi)$ is autocorrelation function of the third Stokes parameter angular distribution. Using the assumption how earlier, we can write

$$
B_{S}^{3}(\Delta \varphi)=\frac{0,5}{2 \pi} \int_{0}^{\infty} \int_{-\pi}^{\pi}(\sqrt{D})^{2} \cos (2 k l \Delta \varphi) W_{1}(\sqrt{D}) d(\sqrt{D}) d \eta=0,5 \bar{D} \cos (2 k l \Delta \varphi),
$$

where $\bar{D}$ is the mean value of elementary scatterers $A$ and $B$ polarization states distance, which was defined by the average of random values $D$ statistical set. The autocovariance function (45) is the interference law of the forth order . A visibility of interference law (45) is defined by polarization coherence degree $\bar{D}=1-\bar{N}$ by virtue of the result (46).

The joint experimental investigation of generalized Fresnel - Arago interference laws in conformity to polarization-energetically properties of two-elements man-made radar objects were realized in the International Research Centre for Telecommunication-Transmission and Radar of TU Delft (Tatarinov et al, 2004). In this subsection we present an insignificant part of these results for the following objects: 1). Two trihedral, where the first was empty and the second was arranged by the elliptic polarizer in the form of special polarization grid. The transmission coefficients along the $O X$ and $O Y$ axes are $b_{Y}=0,5 b_{X}$ and mutual phase shift between polarizer eigen axes is $\varphi_{X Y}=\pi / 2\left(P_{A}=1 ; \dot{P}_{B}=j 0,5\right.$;

$N=0,5 ; \quad D=0,5)$; This object is presented on the fig. 10. 2).Two trihedral, where the first was empty and the second was arranged by the linear polarizer in the form of the special polarization grid. $(N=0,5 ; D=0,5)$;

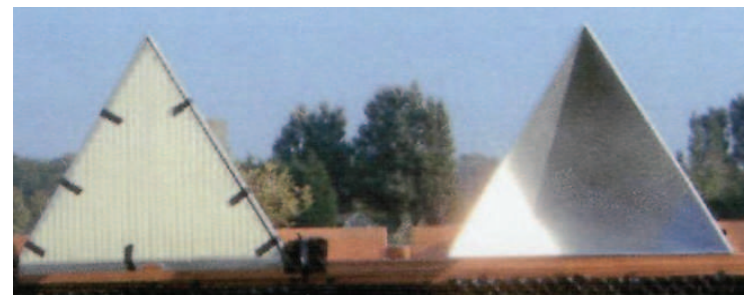

Fig. 10. Two-point radar object N1

The phase centers of the trihedral were distributed in the space on the distance $100 \mathrm{~cm}$, the wave length of the radar was $3 \mathrm{~cm}$. For these parameters the space frequency and space period are $f_{S P}=2 l / \lambda(R a d)^{-1}, T_{S P}=0,015 R a d$ (or $0.855^{0}$ ). The construction, where the trihedral were placed, has rotated with the angular step $0,25^{0}$.

When the object includes the trihedral arranged by the elliptic polarizer and empty trihedral (combination N1), the polarization proximity and distance theoretical estimation is $N=D=0,5$. On the Fig.11a,b the experimental angular harmonics functions (generalized 
interference pictures) $S_{0}(\theta), S_{3}(\theta)$ are shown. It follows from these figures that the visibility for interference picture $S_{0}(\theta)$ is $W_{0} \approx 0,3$ that corresponding to polarization proximity $N_{0}=0.54$ (theoretical estimation is $N=0.5$ ). The visibility for $S_{3}(\theta)$ is $W_{3}=1$ that corresponding to polarization distance $D=0,5$.

For the system including the trihedral arranged by the linear polarizer and empty trihedral (object N2), we can find the theoretical estimation visibility values $W_{0}=0,66 ; W_{3}=1$ that correspond to polarization proximity values $N_{0}=\sqrt{W_{0}}=0,82 ; N_{3}=\sqrt{W_{3}}=1$. On the Fig.12a,b the angular harmonics functions $S_{0}(\theta), S_{3}(\theta)$ for this situation are shown.

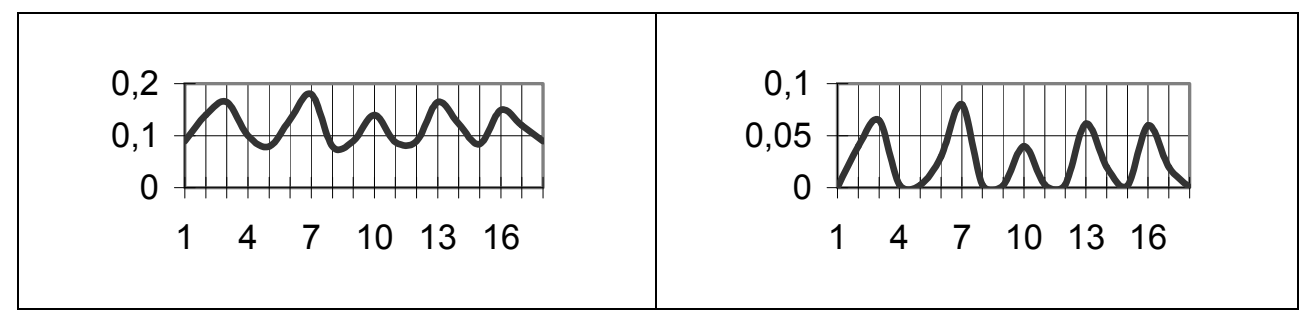

Fig. 11a. Generalized interference law Fig. 11.b. Generalized interference law for the parameter $S_{0}(\theta)$ (object N1) for the parameter $S_{3}(\theta)$ (object N1)

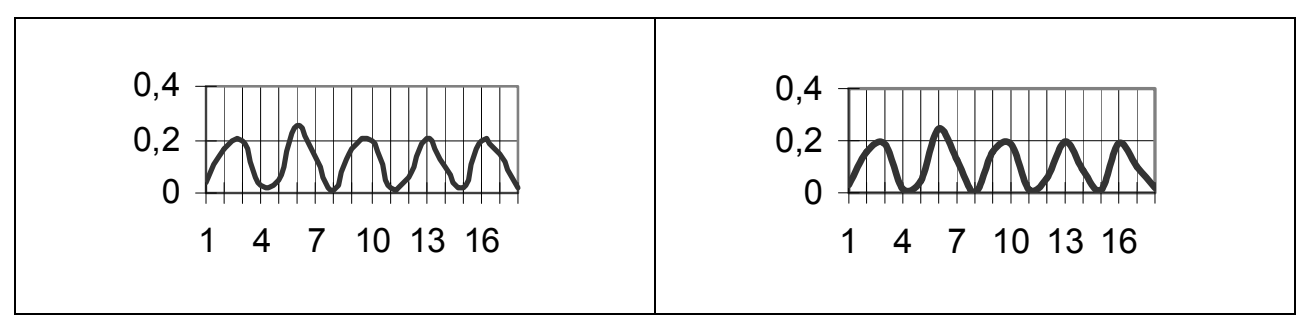

Fig. 12a. Generalized interference law Fig. 12.b. Generalized interference law for the parameter $S_{0}(\theta)$ (object N2) for the parameter $S_{3}(\theta)$ (object N2)

The experimental estimation with the use of Fig.12a,b gives us $N_{0} \approx 0,85 ; N_{3}=1$ what is the satisfactory coinciding with the theoretical estimation.

\section{Polarization - energetic parameters of complex radar object coherent image formation as the interference process. Polarization speckles Statistical analysis}

It is demonstrated in the given subsection that the scattered field polarization-energetically speckles formation at the scattering by multi-point random distributed radar object (RDRO) is the interference process. In this case the polarization-energetic response function of a RDRO can be considered as space harmonics collection. Every space harmonic of this collection will be initiated by one from a great many scattered interference pair, which can be formed by multi-point RCRO scatterers. In this connection every space harmonic will have an amplitude, which will be defined by a value of this pair scatterers polarization states proximity (or distance). As far as the RCRO elementary scatterers positions are stochastic, at the positional angle change and a random number of interference pairs, 
having the same space diversity under the condition of these pair scatterers polarization states proximity stochastic difference, we have the classical stochastic problem. This setting of a problem has been formulated in the first time.

Let's to consider the scattering by a multi-point (complex) radar object (see Fig. 13). For the case of coinciding linear polarization both for transmission and receiving we can write the field scattered by a point $X_{I}$ (RCS of this scatterer is $\sigma_{I}$ ) for some point $Q$ in far zone

$$
\dot{E}_{S}(\theta)=-\frac{\exp \left(j 2 k R_{0}\right)}{R_{0} \sqrt{4 \pi}} \sqrt{\sigma_{I}} \dot{E}_{0} \exp \left(-j 2 k X_{I} \theta\right),
$$

where $R_{I} \approx R_{0}-X_{I} \theta$ is the distance between the scatterers $X_{I}$ and $X ; \dot{E}_{0}$ and $\dot{E}_{S}$ are initial and scattered field electrical vectors respectively. For the case when a scatterers are characterizing by the scattering matrix $\left\|\dot{S}_{I}^{i k}\right\| ;(i, k=1,2)$ then the scattered field complex vector will be connected with initial field complex vector as

$$
\dot{\vec{E}}_{S}(\theta)=-\frac{\exp \left(j 2 k R_{0}\right)}{R_{0} \sqrt{4 \pi}}\left\|\dot{S}_{I}^{i k}\right\| \dot{\vec{E}}_{0} \exp \left(-j 2 k X_{I} \theta\right) .
$$

Let us consider now the electromagnetic field polarization-energetic parameters distribution formation as the interference process at the scattering by multi-point RDRO. For the example we will find that the electrical vector of the field, scattered by 4-points complex object for the case of coinciding linear polarization both for transmission and receiving:

$$
\dot{E}_{S}(\theta)=-\frac{\exp \left(j 2 k R_{0}\right) \dot{E}_{0}}{R_{0} \sqrt{4 \pi}} \sum_{I=1}^{4} \sqrt{\sigma_{I}} \exp \left(-j 2 k X_{I} \theta\right) .
$$

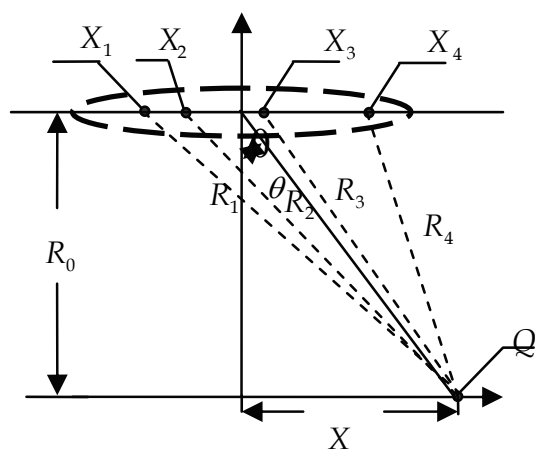

Fig. 13. Waves scattering by multi-point RDRO

Now we can define the instantaneous distribution of scattered field power in the space as the function of the positional angle $\theta$ :

$$
\begin{array}{r}
P(\theta)=\dot{E}_{S}(\theta) \dot{E}_{S}^{*}(\theta)=\sigma_{1}+\sigma_{2}+\sigma_{3}+\sigma_{4}+2 \sqrt{\sigma_{1} \sigma_{2}} \cos \left(2 k d_{12} \theta\right)+2 \sqrt{\sigma_{1} \sigma_{3}} \cos \left(2 k d_{13} \theta\right)+ \\
+2 \sqrt{\sigma_{1} \sigma_{4}} \cos \left(2 k d_{14} \theta\right)+2 \sqrt{\sigma_{2} \sigma_{3}} \cos \left(2 k d_{23} \theta\right)+2 \sqrt{\sigma_{2} \sigma_{4}} \cos \left(2 k d_{24} \theta\right)+2 \sqrt{\sigma_{3} \sigma_{4}} \cos \left(2 k d_{34} \theta\right) .
\end{array}
$$


So, the instantaneous distribution of scattered field power in the space as the function of the positional angle $\theta$ is formed by the union of elementary scatterer radar cross section (4 items) plus 6 cosine oscillations. It is not difficult to see that every cosine functions are caused by the interference effect between the fields scattered by a pair of elementary scatterers forming the RCRO. The number of this pairs can be found with the use binomial coefficient

$$
C_{M}^{N}=M ! /[N !(M-N) !],
$$

where $M$ is a number of values, $N$ is a number elements in the combination. In the case when $M=4, N=2$, we have $C_{4}^{2}=6$. So, the angular response function of the complex radar object considered will include 6 space harmonic functions as the interference result summarize how it follows from the expression (48) where the values $d_{12}=X_{1}-X_{2}$; $d_{13}=X_{1}-X_{3} ; d_{14}=X_{1}-X_{4} ; d_{23}=X_{2}-X_{3} ; d_{24}=X_{2}-X_{4} ; d_{34}=X_{3}-X_{4}$ are the space diversity of scattered elements for every interference pair. The space harmonic function $\sqrt{\sigma_{i} \sigma_{k}} \cos \left(2 k d_{i k} \theta\right)$ corresponds to the definition that was done in (Kobak, 1975), (Tatarinov et al, 2007) . In accordance with this definition, the harmonic oscillation in the space having the type $\cos (2 k d \theta)$ is defined by the full phase $\psi(\theta)=2 k d \theta=(2 \pi / \lambda) 2 d \theta$, the derivative from which is the space frequency $f_{S P}=2 d / \lambda$ having the dimension $\mathrm{Rad}^{-1}$. The period $T_{S P}=1 / f_{S P}=\lambda / 2 d$ has the dimension $R a d$, which corresponds to this frequency.

So, a full power distribution of the field, scattered by complex radar object, is an union of the interference pictures, which are formed by a collection of elementary two-points interferometers.

Thus, we can write a scattered power random angular representation, depending on the positional angle, in the form

$$
P(\theta)=\sum_{m=1}^{M} \sigma_{m}^{2}+2 \sum_{1}^{C} \sqrt{\sigma_{i} \sigma_{k}} \cos \left(2 k d_{i k} \theta\right),
$$

where $C=C_{M}^{2}$ is combinations number, $M$ is a full number of RCRO elementary scatterers. It was demonstrated above that the electromagnetic field Stokes parameter $S_{0}, S_{3}$ angular distribution at the scattering by two-point distributed object has the form

$$
S_{0}(\theta)=S_{0}^{a}+S_{0}^{b}+2 \sqrt{S_{0}^{a}} \sqrt{S_{0}^{b}} \sqrt{N_{a b}} \cos (\xi+0,5 \varphi) ; S_{3}(\theta)=S_{3}^{a}+S_{3}^{b}+2 \sqrt{S_{0}^{a}} \sqrt{S_{0}^{b}} \sqrt{D_{a b}} \cos (\xi-0,5 \varphi),
$$

where $\xi=2 k l \theta$. It follows from this expression that the space harmonics functions $\cos (2 k l \theta \pm \eta)$ are having amplitudes $\sqrt{S_{0}^{a}} \sqrt{S_{0}^{b}} \sqrt{N_{a b}}$ or $\sqrt{S_{0}^{a}} \sqrt{S_{0}^{b}} \sqrt{D_{a b}}$. Here the values $N_{a b}, D_{a b}$ are a proximity (distance) of distributed object elementary scatterers polarization states respectively.

Taking into account above mentioned, we can write the Stokes parameters angular distribution for the field, scattered by random complex radar object as an union of the generalized interference pictures, which are formed by a collection of elementary two-points interferometers (see Fig.13):

$$
S_{0}(\theta)=\sum_{m=1}^{M} S_{0}^{m}+2 \sum_{1}^{C} \sqrt{S_{0 i} S_{0 k}} \sqrt{N_{i k}} \cos \left(\xi_{i k}+\eta_{i k}\right),
$$




$$
S_{3}(\theta)=\sum_{m=1}^{M} S_{3}^{m}+2 \sum_{1}^{C} \sqrt{S_{0 i} S_{0 k}} \sqrt{D_{i k}} \cos \left(\xi_{i k}+\eta_{i k}\right),
$$

where $C=C_{M}^{2}$ is combinations number. An amplitude of every space harmonics and initial space phases of these harmonics will be stochastic values and the further analysis must be statistical. First of all we will find a theoretical form of scattered field Stokes parameter $S_{3}$ angular distribution autocorrelation function. As far as we would like to find the autocorrelation function (not covariance function!), we must eliminate a random constant item $\sum_{m=1}^{M} S_{3}^{m}$ from the stochastic function $S_{3}(\theta)$ for the guarantee of zero mean value. Taking into account that the value $\sum_{m=1}^{M} S_{3}^{m}$ can be as no stationary stochastic function, the average must be made using a sliding window. After a mean value elimination and normalization we can write stochastic stationary function $S_{3}(\theta)$ in the form

$$
S_{3}(\theta)=\sum_{1}^{C} \sqrt{D_{i k}} \cos \left(2 k d_{i k} \theta+\eta_{i k}\right)
$$

Its autocorrelation function can be found as

$$
B_{S}(\Delta \theta)=\sum_{N=1}^{C} \int_{-\infty}^{\infty} \int_{-\infty}^{\infty}\left(\sqrt{D_{N}}\right)^{2} \cos \left[2 k d_{N} \theta+\eta\right] \cos \left[2 k d_{N}(\theta+\Delta \theta)+\eta\right] W_{2}(\sqrt{D}, \eta) d(\sqrt{D}) d \eta .
$$

Here amplitudes $\sqrt{D}$ and space initial phase $\eta$ of space harmonics are random values, which can be characterized by two-dimensional probability distribution density $W_{2}(\sqrt{D}, \eta)$, and $\Delta \theta=\theta_{1}-\theta_{2}$. We will suppose that random amplitudes and phases are independent variables. For this case two-dimensional probability distribution can be presented as two one-dimensional distributions densities product

$$
W_{2}(\sqrt{D}, \eta)=W_{1}(\sqrt{D}) W_{1}(\eta) \cdot
$$

Let's suppose also that random phase has the uniform probability distribution density on the interval $(-\pi, \pi)$ i.e. $W(\eta)=1 / 2 \pi$. A probability distribution density for the random amplitude $\sqrt{D}$ can be preassigned, however for all cases it will be one-sided. After the integration we obtain the value of double integral in the form

$$
I=\frac{0,5}{2 \pi} \int_{0}^{\infty} \int_{-\pi}^{\pi}\left(\sqrt{D_{N}}\right)^{2} \cos \left(2 k d_{N} \Delta \theta\right) W_{1}(\sqrt{D}) d(\sqrt{D}) d \eta=0,5<D_{N}>\cos \left(2 k d_{N} \Delta \theta\right),
$$

where $\left\langle D_{N}\right\rangle$ is the polarization distance mean value, which was found by the average along the statistical ensemble of random values $D_{N}$ for all space harmonics having the space frequency $f_{S P}^{N}=2 d_{N} / \lambda$. Thus, we can write the theoretical form of scattered field Stokes parameter angular distribution autocorrelation function in the form

$$
B_{S}(\Delta \theta)=0,5 \sum_{N=1}^{C}<D_{N}>\cos \left(2 k d_{N} \Delta \theta\right)
$$


Taking into account that the every item of the union (51) is the autocorrelation function for an isolated space harmonic oscillation $S_{N}(\theta)=\sqrt{D_{N}} \cos \left(2 k d_{N} \theta+\eta_{N}\right)$ having random amplitude $\sqrt{D_{N}}$ and random initial space phase $\eta_{N}$, i.e.

$$
B_{S N}(\Delta \theta)=0,5<D_{N}>\cos \left(2 k d_{N} \Delta \theta\right)
$$

it is not difficult to see that the autocorrelation function of the Stokes parameter stochastic realization is the union of individual autocorrelation functions of all space harmonics:

$$
B_{S}(\Delta \theta)=\sum_{N=1}^{C} B_{S N}(\Delta \theta) .
$$

Let's now to find a complex radar object averaged space spectra using the expressions (8) for polarization-angular response autocorrelation function. The power spectra for the case of isolated space harmonic can be found as the Fourier transformation above the autocorrelation function (52):

$$
P\left(\Omega_{S P}\right)=\int_{-\infty}^{\infty} B_{S N}(\Delta \theta) \exp \left(-j \Omega_{S P} \Delta \theta\right) d(\Delta \theta)=0.5<D_{N}>\left[\delta\left(\Omega_{S P}-\Omega_{S P}^{N}\right)+\delta\left(\Omega_{S P}+\Omega_{S P}^{N}\right)\right],
$$

where $\Omega_{S P}=2 \pi f_{S P}=2 \pi(2 d / \lambda)$ is a space frequency. The spectra lines are placed on the distances $\pm \Omega_{S P}^{N}$ from the co-ordinates system origin and their positions are defined by the space frequency $f_{S P}^{N}=2 d_{N} / \lambda$ of two-point radar object. This space frequency is corresponding to space diversity of two reflectors distributed in the space. The intensity of power spectra lines is determined by polarization distance between polarization states of two scatterers forming the radar object.

The full space spectra of stochastic polarization-angular response, i.e. Fourier transformation of the autocorrelation function (53) is:

$$
P\left(\Omega_{S P}\right)=0,5 \sum_{N=1}^{C}<D_{N}>\left[\delta\left(-\Omega_{S P}^{N}\right)+\delta\left(+\Omega_{S P}^{N}\right)\right]
$$

It is necessary to indicate here that a connection between scattered (diffracted) field polarization parameters and polarization parameters distribution along a scattering (diffracting) object in the form of Fourier transformation pair is established in the first time. However, this connection is correct for fourth statistical moments: scattered field intensity correlations (include mutual intensity) and polarization proximity (distance) distribution along a scattering (diffracting) object.

In the conclusion we consider some results of scattered field polarization parameters investigation at the scattering by random distributed object having a lot of scattering centers - "bright" points. It follows also both from theoretical and experimental investigations results that polarization-angular response function of a RCRO in the form of the 3-rd Stokes parameter angular dependence corresponds to a narrow-band random process. The experimental realization of this parameter has shown on the fig.7. The angular interval for this dependence is $\pm 20^{0}$. The rotated caterpillar vehicle (the sizes $5,5 \times 2,5 \times 1,5 \mathrm{~m}$ ) placed on the distance $2 \mathrm{~km}$ was used as complex radar object. The autocorrelation functions (ACF) of 
this object response $S_{3}(\Delta \theta)$ are shown on the fig.14. The ACF on the angular interval $\pm 20^{0}$ concerning the direction to the object board is designated by dotted line and the ACF into the same interval in direction to the stern of the object is continue line. The measurements in these directions allow us to take into account the difference in the radar object space spectra band at its observation in areas of perpendiculars to the board and to the stern of the object. On the fig. 15 RDRO mean power spectra are shown. Dotted line is corresponding to direction to the object board and continue line corresponds to object stern.

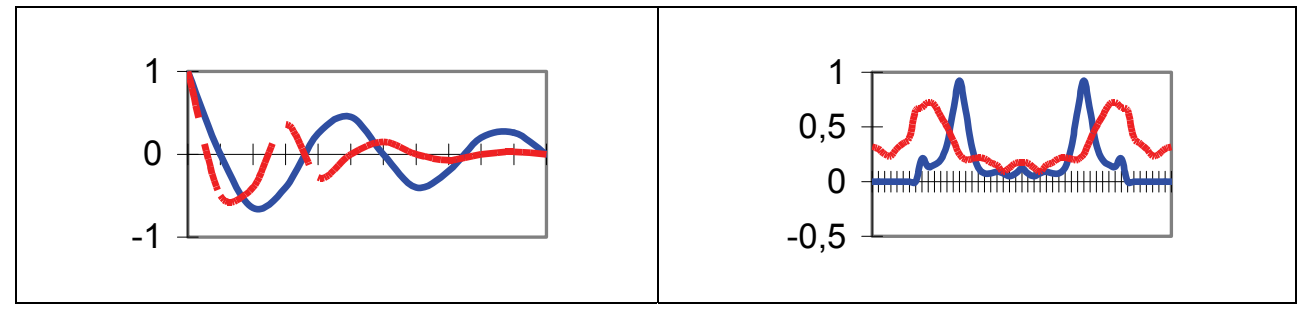

Fig. 14. Autocorrelation functions of RDRO Fig.15. Mean power space spectra of RDRO stochastic polarization-angular response

\section{Conclusion}

In the conclusion we can to indicate that in the Chapter proposed a new statistical theory of distributed object polarization speckles (coherent images) has been developed. The use of fourth statistical moments and emergence principle allow us to find the answers for a series of problems which are having the place at the electromagnetic waves coherent scattering by distributed (complex) radar objects.

\section{References}

Proceedings of the IEEE. (1965). Special issue. Vol. 53., No.8, (August 1965)

Ufimtsev, P. (1963). A Method of Edge Waves in Physical Diffraction Theory, Soviet Radio Pub. House, Moscow, Russia

Proceedings of the IEEE. (1989). Special issue. Vol. 77., No.5, (May 1965)

IEEE Transaction on Antennas and Propagation . (1989). Special issue. No.5, (May 1965)

Ostrovitjanov, R. \& Basalov F. (1982). A Statistical Theory of Distributed Objects Radar, Radio and Communication Pub. House, Moscow, Russia

Shtager, E. (1986). Waves Scattering by Complicated Radar Objects, Radio and Communication Pub. House, Moscow, Russia

Kell, R. (1965). On the derivation of bistatic RCS from monostatic measurements. Proceedings of the IEEE, Vol. 53, No. 5, (May 1965), pp 983-988

Stratton, J. \& Chu, L. (1939). Diffraction theory of electromagnetic waves. Phys. Rev., Vol. 56, pp 308-316

Tatarinov, V. ; Tatarinov S. \& Ligthart L. (2006). An Introduction to Radar Signals Polarization Modern Theory (Vol. 1: Plane Electromagnetic Waves Polarization and its Transformations), Tomsk State University Publ. House, ISBN 5-7511-1995-5, Tomsk, Russia 
Shtager, E. (1994). Radar objects characteristics calculation at random earth and sea surface. Foreign Radioelectronics, No. 4-5, (May 1994), pp 22-40, Russia

Steinberg, B. (1989). Experimental localized radar cross section of aircraft. Proceedings of the IEEE, Vol. 77, No. 5, (May 1989), pp 663-669

Kobak, V. (1975). Radar Reflectors, Soviet Radio Pub. House, Moscow, Russia

Kanareikin, D.; Pavlov, N. \& Potekchin V. (1966). Radar Signals Polarization, Soviet Radio Pub. House, Moscow, Russia

Pozdniak, S. \& Melititsky V. (1974). An Introduction to Radio Waves Polarization Statistical Theory, Soviet Radio Pub. House, Moscow, Russia

Franson, M. (1980). Optic of Speckles. Nauka Pub. House, Moscow, Russia

Peregudov, F. \& Tarasenko, F. (2001). The Principles of Systems Analysis, Tomsk State University Publ. House, Tomsk, Russia

Azzam, R. \& Bashara, N. (1977). The Ellipsometry and Polarized Light, North Holland Pub. House, New York-Toronto-London

Tatarinov, V. ; Tatarinov, S. \& van Genderen P. (2004). A Generalized Theory on Radar Signals Polarization in Space, Frequency and Time Domains for Scattering by Random Complex Objects. Report of IRCTR-S-004-04, Delft Technology University, the Netherlands

Born, M. \& Wolf, E. (1959). Principles of Optics. Pergamon Press, New-York-Toronto-London Potekchin, V. \& Tatarinov, V. (1978). The Coherence Theory of Electromagnetic Fielg, Svjaz Pub. House, Moskow, Russia

Tatarinov, V. ; Tatarinov S. \& Kozlov, A. (2007). An Introduction to Radar Signals Polarization Modern Theory (Vol. 2: A Statistical Theory of Electromagnetic Field ), Tomsk State University Publ. House, ISBN 978-5-86889-476-3, Tomsk, Russia 


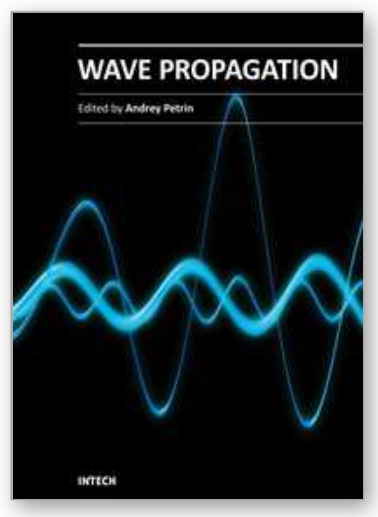

\author{
Wave Propagation \\ Edited by Dr. Andrey Petrin
}

ISBN 978-953-307-275-3

Hard cover, 570 pages

Publisher InTech

Published online 16, March, 2011

Published in print edition March, 2011

The book collects original and innovative research studies of the experienced and actively working scientists in the field of wave propagation which produced new methods in this area of research and obtained new and important results. Every chapter of this book is the result of the authors achieved in the particular field of research. The themes of the studies vary from investigation on modern applications such as metamaterials, photonic crystals and nanofocusing of light to the traditional engineering applications of electrodynamics such as antennas, waveguides and radar investigations.

\title{
How to reference
}

In order to correctly reference this scholarly work, feel free to copy and paste the following:

Victor Tatarinov and Sergey Tatarinov (2011). A Statistical Theory of the Electromagnetic Field Polarization Parameters at the Scattering by Distributed Radar Objects, Wave Propagation, Dr. Andrey Petrin (Ed.), ISBN: 978-953-307-275-3, InTech, Available from: http://www.intechopen.com/books/wave-propagation/a-statisticaltheory-of-the-electromagnetic-field-polarization-parameters-at-the-scattering-by-distr

\section{INTECH}

open science | open minds

\section{InTech Europe}

University Campus STeP Ri

Slavka Krautzeka 83/A

51000 Rijeka, Croatia

Phone: +385 (51) 770447

Fax: +385 (51) 686166

www.intechopen.com

\section{InTech China}

Unit 405, Office Block, Hotel Equatorial Shanghai

No.65, Yan An Road (West), Shanghai, 200040, China

中国上海市延安西路65号上海国际贵都大饭店办公楼405单元

Phone: +86-21-62489820

Fax: +86-21-62489821 
(C) 2011 The Author(s). Licensee IntechOpen. This chapter is distributed under the terms of the Creative Commons Attribution-NonCommercialShareAlike-3.0 License, which permits use, distribution and reproduction for non-commercial purposes, provided the original is properly cited and derivative works building on this content are distributed under the same license. 